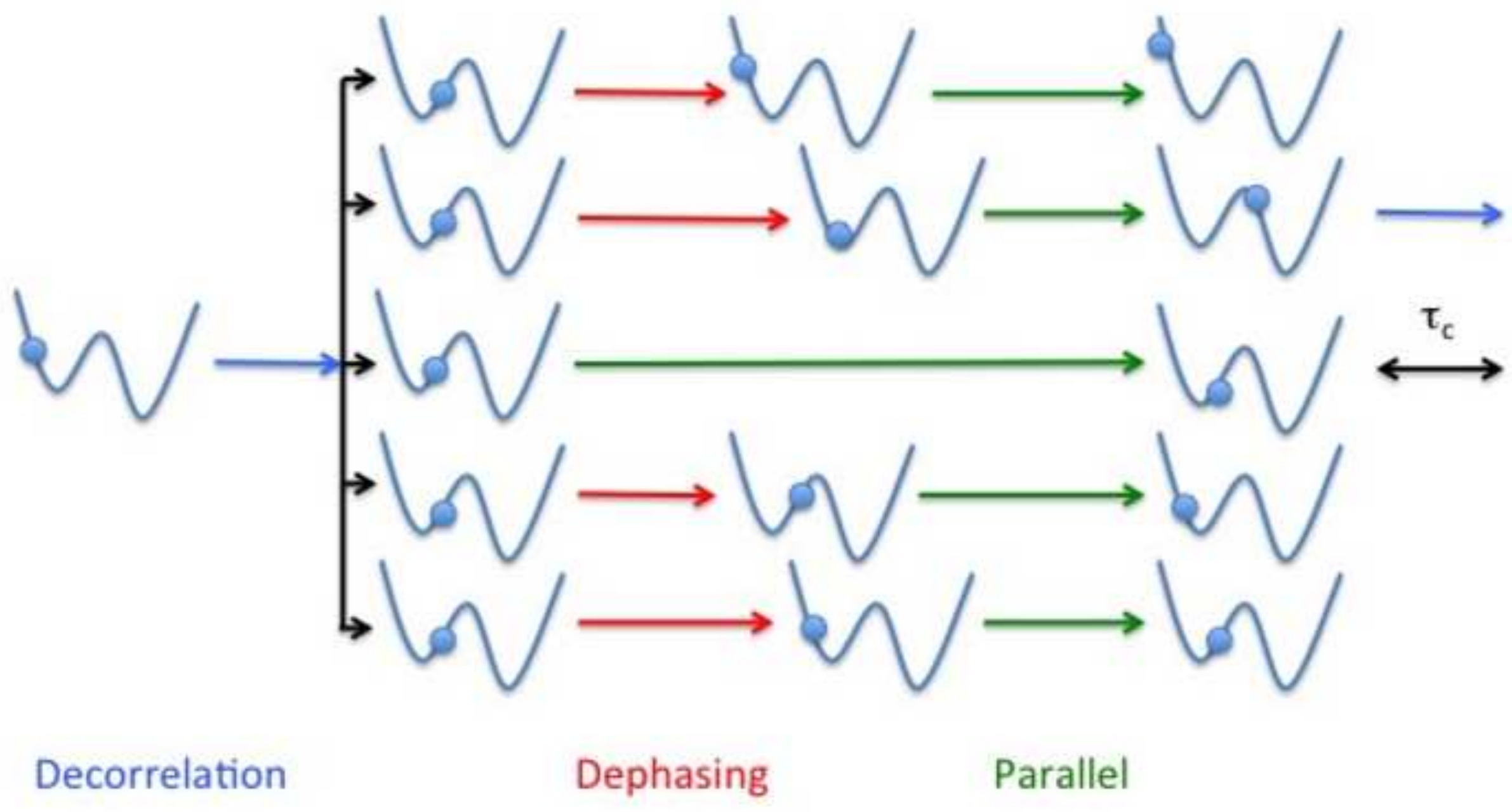




\title{
The parallel replica dynamics method -- coming of age
}

\author{
Danny Perez ${ }^{1, *}$ \\ Blas P. Uberuaga ${ }^{2}$ \\ Arthur F. Voter ${ }^{1}$ \\ ${ }^{1}$ Theoretical Division T-1, Los Alamos National Laboratory, Los Alamos, NM, 87545, USA \\ ${ }^{2}$ Materials Science and Technology Division MST-8, Los Alamos National Laboratory, Los \\ Alamos, New Mexico 87545, USA \\ *email: danny perez@lanl.gov
}

\begin{abstract}
Molecular Dynamics (MD) --- the numerical integration of atomistic equations of motion --- is a workhorse of computational materials science. Indeed, MD can in principle be used to obtain any thermodynamic or kinetic quantity, without introducing approximation or assumptions beyond the adequacy of the interaction potential. It is therefore an extremely powerful and flexible tool to study materials with atomistic spatio-temporal resolution. These enviable qualities however come at a steep computational price, limiting the system sizes and simulation times that can be achieved in practice. While the size limitation can be efficiently addressed with massively parallel implementations of MD based on spatial decomposition strategies, allowing for the simulation of trillions of atoms, the same approach usually cannot extend the timescales much beyond microseconds. In this article, we discuss an alternative, parallel-in-time, strategy -the Parallel Replica Dynamics (ParRep) method -- that aims at addressing the timescale limitation of MD for systems that evolve through rare state-to-state transitions. We review the formal underpinnings of the method, including recent developments showing it can provide arbitrarily accurate results for any definition of the states. When an adequate definition of the states is available, ParRep can simulate trajectories with a parallel speedup approaching the number of replicas used. We demonstrate the usefulness of ParRep by presenting different examples of materials simulations where access to long timescales was essential to study the physical regime of interest and discuss practical considerations that must be addressed to carry out these simulations. Sixteen years after its introduction, with a new understanding of its generality and ever increasing availability of parallel processing, the ParRep method is coming of age.
\end{abstract}

Keywords: Computer Simulations; Molecular Dynamics; Accelerated Molecular Dynamics; Parallel Replica Dynamics; 


\section{Introduction}

Molecular dynamics (MD), which simply consists of numerically solving the equations of motion for a set of atoms, is one of the cornerstones of computational materials science. MD can in principle be used to obtain any thermodynamic or kinetic quantity, without introducing approximations beyond the assumption of classical mechanics and the adequacy of the interaction potential. It can be used to elucidate atomistic structures and mechanisms that aid in the interpretation of experiment, or even reveal behavior that is unavailable to experiment. For example, almost everything we know about the production of defects during collision cascades in radiation damage environments comes from MD simulations (interestingly, one of the first applications of MD, probably the first using a continuous potential, was precisely to study collision cascades [1]). Full atomistic resolution comes at a cost, however; direct MD simulation is limited in the length and time scales it can achieve. The length scale limitation can be overcome to some extent by using spatial decomposition on a parallel computer, an approach developed over the past 20-25 years, as illustrated in the center panel of Fig. 1. At present, simulations of millions of atoms are common, billions of atoms can be evolved when needed, and it is possible to perform simulations of up to about a trillion atoms, corresponding to a few cubic microns of material. This accessible size will continue to grow as computer architectures become increasingly more parallel. The time-scale problem, however, is much more daunting. The sequential nature of the time integration, with a time step that is typically a few fs, limits the total simulation time to a few microseconds. For example, a system with just 1000 atoms, described with a short-ranged empirical potential such as the embedded atom method [2], evolved for a week on a state-of-the-art processor, will reach a time of just over one microsecond. Parallelizing this system using spatial decomposition offers only an incremental improvement: the spatial domains are ineffectively small and the computation becomes communication bound. Similarly, a graphical processing unit (GPU) can offer a spatial parallelization speedup of an order of magnitude or more, but their efficiency suffers for small systems. Moreover, anticipated advances in computer architecture in the coming years will give ever-greater levels of parallelization, but essentially no improvement (and perhaps even reductions) in per-processor speed. For biomolecular simulations, specialized hardware has extended this the time scale limit by a couple of orders of magnitude [3]; this is a significant advance, but at present no such hardware exists for materials simulations. One can of course use a parallel computer to run many independent trajectories (see left panel of Fig. 1), but it can be difficult to use these short segments to infer long-time behavior. Consequently, for the foreseeable future, direct MD simulations of materials are limited to microseconds, putting direct comparison with experimentally relevant conditions well out of reach for many technologically important processes, such as film growth, radiation damage annealing, plastic flow, or corrosion. When more computationally expensive interatomic potentials are employed, this is reduced to nanoseconds, and for MD based on forces from electronic-structure theory (e.g., density functional theory or quantum chemistry), the time-scale limit is on the order of picoseconds. 
X event

M simulations of size $\mathrm{N}$ for time $\mathrm{t}$

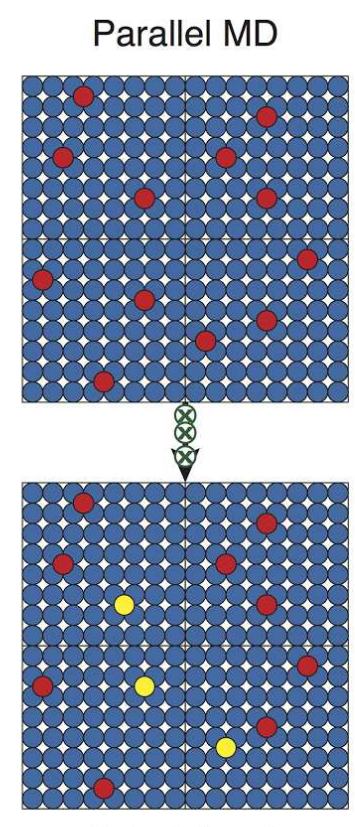

1 simulation of size MN for time $t$

\section{ParRep}

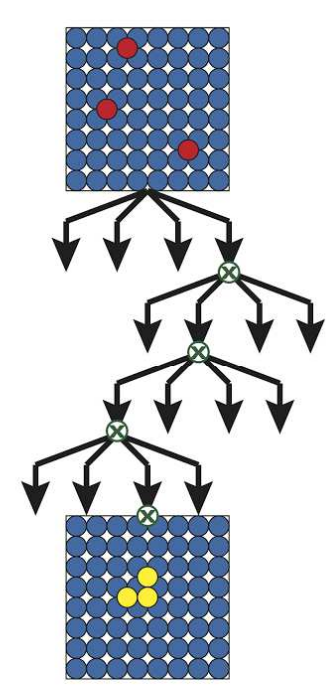

1 simulation of size $\mathrm{N}$ for time $\mathrm{Mt}$

Figure 1: Illustration of the different parallelization strategies for MD for adatoms (red) on a surface (blue). Left: independent simulations; Center: spatial parallelization; Right: ParRep. Time flows from top to bottom, as indicated by the arrows. Adatoms are drawn in yellow when they moved from their initial position. Events that take the system from one state to another are schematically indicated by ' $x$ '.

Although direct MD has a severely limited time scale, a common characteristic of many materials systems offers a path for simulating longer times. For the majority of systems of interest in materials science (as well as in chemistry, biology, and many other fields), the dynamical evolution on long timescales consists of infrequent transitions from one state of the system to another. During the time between these transitions, the system may remain in a single basin of the potential surface, executing only thermal vibrations. The definition of a state may be more general than this, as we will discuss below, but for now, we will assume this single-basin picture. A transition between states thus involves the passage over a ridge-top in the many-dimensional ( $3 \mathrm{~N}$, where $\mathrm{N}$ is the number of moving atoms) space to another potential basin. For this type of infrequent-event system, the challenge of evolving a system to long times can thus be re-expressed in the following way: From the current state of the system, we ask when the next transition will occur, and to what new potential basin the system will jump. If we can answer these two questions accurately, repeating for each new state the system visits, then we have accurately coarse-grained the time evolution. If these determinations can be performed more efficiently than with direct MD, then this type of approach can be used to reach much longer times than are accessible with direct MD. 
Simulation methods in this general class have their roots in the 1960s and 1970s, with the development of a methodology that later became known as kinetic Monte Carlo (KMC) (see Ref. [4]). Briefly, in KMC, one first specifies a set of states available to the system, often corresponding to possible occupations on the native crystal lattice, and then estimates, or computes, the rate constant for all transitions that connect these states. Given this set of states and rate constants, $\mathrm{KMC}$ provides a stochastic procedure $[5,6]$ for appropriately choosing the next transition, and the time for that transition. The essence of the KMC algorithm is extremely simple: after entering a state, we choose a time for the next transition from the normalized exponential distribution $p(t)=k e^{-k t}$, where $k$ is the sum of the rate constants for escape from this state, and then we choose one of the possible transition paths out of this state with a probability proportional to the rate constant for that path. Repeating this simple procedure gives a state-to-state trajectory that is correct, representing a valid realization of the state-to-state dynamics, for this set of Markovian states and the first-order rate constants connecting them. An appealing property of the KMC approach is that if our state-specification procedure can generate a complete list of the states (potential basins) of the system, if we know all possible escape paths from each of these states, and if we know the rate constant accurately for each of these escape paths, then the state-to-state trajectory generated by a KMC simulation will accurately represent the state-to-state trajectory generated by a molecular dynamics simulation on that potential surface. In the limit that the states are truly Markovian and the rate constants are exact, then this correspondence would be exact. In practice, the rate constants can be computed with transition state theory (TST) [7-9], and typically the harmonic approximation to TST [10] is employed. If desired, the TST rates can be corrected for correlated dynamical events as well $[11,12]$.

The problem with $\mathrm{KMC}$ in this form is that the list of possible transitions out of each state is typically incomplete. Transitions may take the system off lattice, and even for on-lattice states there can be surprisingly complicated and perhaps important mechanisms that take the system between these states. For example, for metal surface-diffusion systems, this became clear in the early 1990s when two-atom exchange events were discovered to be important even on extremely simple surfaces $[13,14,15]$ where hop mechanisms alone had been previously assumed, and soon highly concerted mechanisms were understood to be important in many situations [16-19] important to surface evolution and film or crystal growth. Such events show up regularly in the bulk as well [20-25].

A way to improve on simple lattice-based KMC is to generate, on the fly, a more complete list of transition paths out of the current potential basin, by searching for saddle points surrounding the state. The escape rate for each of these pathways is then computed, typically using HTST or an approximation to it. Although much more computationally intensive, this approach, now typically referred to as adaptive KMC (AKMC) [26-28] can be very powerful. AKMC finds concerted events automatically if they exist, and also allows the system to go off lattice if that is where a transition path takes it. The main challenge in AKMC becomes efficiently finding a list of saddles that is complete enough to give accurate dynamics. It probably cannot be proven that all the saddles have been found, although there have been noteworthy advances in assigning approximate error bounds [29]. 
An alternative approach to the infrequent event problem is the accelerated molecular dynamics (AMD) concept. In AMD methods, rather than trying to find all possible escape pathways from the current state of the system, we instead try to find a single escape path, the path that is appropriate for the next escape. Just as a very long MD trajectory finds its own way out of each state with no prior knowledge of the possible escape paths, the idea in AMD is to design an MDbased algorithm that causes the trajectory to discover its escape path more quickly, introducing as little error as possible into the way it makes this choice. A few methods in this class have been developed, beginning in the late 1990s, namely hyperdynamics [30], parallel replica dynamics [31], and temperature accelerated dynamics [32]. A closely related and powerful approach, which has some characteristics of both AMD and AKMC, is kappa-dynamics [33].

In the hyperdynamics method $[30,34]$, the potential energy surface is augmented with a bias potential that tends to fill in the potential basins, thereby making the escape barriers lower and the rates faster. If the bias potential $\Delta V$ is constructed so that $\Delta V=0$ along all the ridgetops between states, and if the system before and after adding the bias has no TST-violating correlated events, it can be shown that the system will advance from state to state in a correct way, with an accelerated time estimate (the hypertime) that is evaluated on the fly and has unbiased statistical errors. Designing a bias potential that can achieve these requirements without prior knowledge of the reaction mechanisms in the system is a substantial challenge and the subject of ongoing research. Nonetheless, very powerful bias forms have been proposed, and with a good bias potential the computational speedup relative to direct MD, what we term the boost factor, can be many orders of magnitude. One very successful form is the bond-boost bias potential of Miron and Fichthorn [35].

In parallel-replica dynamics (ParRep) [31], the trajectory escape path is found more quickly by simultaneously running many replicas of the system. Perhaps surprisingly, and this will be derived and discussed in detail below, arbitrarily accurate dynamics are obtained simply by taking the transition to be the first escape found on any of the replicas, and taking the escape time to be the sum of MD times on all the replicas up to the instant of that escape.

In the temperature accelerated dynamics (TAD) [32] method, the escape is hastened by raising the temperature of the system from the intended temperature $T$ to a higher temperature $T_{\text {high }}$. The first escape at this higher temperature will not necessarily be an appropriate one, as the rates for high-barrier processes are increased more than for low-barrier processes. Thus, when this escape is observed, the trajectory is replaced in the original basin, rethermalized at $T_{\text {high }}$, and continued until another escape is observed, and this procedure is repeated. Because within the HTST approximation the temperature dependence is purely Arrhenius $(k=$ $v_{0} e^{-E / k_{B} T}$, each of the escape times at $T_{\text {high }}$ can be converted to an escape time at $T$ once the energy barrier $E$ is known. With an additional assumption that all prefactors $v_{0}$ in the system are above some lower-bound value (e.g., $10^{11} \mathrm{~s}^{-1}$ ), it is then possible to determine when the hightemperature trajectory can be terminated, knowing with a desired confidence that one of the events already observed is the event that would have occurred first at low temperature $T$ in this 
realization of the dynamics. This typically requires that a few transitions be observed at $T_{\text {high }}$. At the cost of some additional approximations (in particular, that HTST applies and that there is a lower bound on $v_{0}$ ), the TAD method offers exponential boost on a single processor, similar to hyperdynamics, but without the requirement that a bias potential be constructed. As with all of the AMD methods, when the lowest barrier is high, the boost factor can be many orders of magnitude. Various enhancements on this basic algorithm have also been proposed [36-40].

These AMD methods have different characteristics and offer different levels of accuracy, more detailed discussion of which can be found in the original papers as well as in review articles [4142]. They have proven to be powerful for a wide range of material and chemical systems, ranging in character from bulk to surface to nanostructural to gas-phase, and they have been implemented into some standard MD simulation packages [43-45]. They have even found some application in non-atomistic simulations, such as computer vision [46]. Further development of these methods to make them more powerful and generally applicable is ongoing, especially to increase their robustness in the presence of low barriers, their computational scaling with system size, and their efficiency in increasingly parallel environments.

In this article, we offer an introduction and review of just one of these AMD methods, the ParRep method. We have chosen to focus on ParRep for three reasons: First, the general availability of parallel computing power has grown substantially since the ParRep method was originally introduced. Consequently, in addition to being the most accurate AMD approach, ParRep is increasingly likely to be the most powerful choice as well, a trend that is likely to persist for the foreseeable future. Second, ParRep is extremely flexible -- it can be used to treat some types of systems that are not amenable to the other AMD methods, or the AKMC methods, due to the assumptions those methods make or the way they operate. One example is a system that is trapped in a state by an entropic bottleneck rather than an energetic barrier. Third, a recent mathematical development [47] has shown that ParRep is even more general than was originally recognized. For overdamped Langevin dynamics and probably for many other types of dynamics as well, it can be shown that ParRep gives arbitrarily accurate dynamical evolution of the system, even when the states are defined without regard to whether they are Markovian. This results from the fact that the ParRep dephasing procedure, carried out for a sufficiently long time, prepares a distribution of trajectory points that behave in an approximately Markovian way, with an exponential distribution of first-passage times, as needed for the ParRep parallel stage. We believe the consequences of this new understanding will be far-reaching, allowing accurate treatment of much more complex systems where a natural state definition involves lumping states together or where it is not even clear at the outset what constitutes a good state definition. We envision this being powerful for complex materials, biochemical systems, soft-matter systems, and even some non-atomistic systems. We are just beginning to exploit this new capability. Sixteen years after its introduction, the ParRep method appears to be coming of age.

The paper is organized as follows: we first lay down the theoretical framework within which ParRep can be understood and discuss its accuracy and efficiency under various assumptions; second, we provide examples of successful use of ParRep for materials simulations, with an 
emphasis on illustrating the breadth of systems that be treated and the practical considerations that must be faced in order to carry out these simulations; finally, we review some implementation details that can be used to improve the efficiency of ParRep simulations, before concluding.

\section{Theoretical Foundation of ParRep}

We begin by reviewing the theoretical underpinnings of ParRep. After describing the algorithm, we explore its formal justification in different settings, ranging from Markovian to general nonMarkovian state-to-state dynamics. We then discuss its accuracy and efficiency and review certain considerations related to the optimal definition of states.

\section{The ParRep Algorithm}

Consider a system whose phase space is partitioned into a set of discrete states. We will show later that these states can be arbitrarily defined, but to develop the ideas, one might adopt a convenient (and conventional) definition where states correspond to basins of attraction of local minima of the potential energy surface. The goal is to generate a statistically proper state-tostate trajectory, i.e., a sequence of states and residence times that provides a coarse description of a single atomistic trajectory. Assume the availability of $N_{r}$ computing units that are each capable of carrying out MD simulations; these are the replicas. The ParRep algorithm, illustrated in Fig. 2, then proceeds as follows. Starting from a given initial state, let a single replica carry out an MD simulation until it spends a time $\tau_{c}$ (the so-called correlation time, an adjustable parameter of the method) without leaving a given (but not necessarily the initial) state. This is the decorrelation stage (blue). At this point, broadcast the current configuration of the system to all other replicas (black arrows). Each one of these additional replicas then proceeds to the dephasing stage, running an MD trajectory until it remains for $\tau_{c}$ in the initial state (red). If a trajectory escapes before that time, the system is placed back in the initial state and that replica reattempts the dephasing stage once more. Coupled with the randomization of the momenta and/or the use of a stochastic thermostat, this has the effect of making the replicas statistically independent. After successfully dephasing and decorrelating, replicas enter the parallel stage (green), where they independently carry out MD until any one of them observes a transition to a new state (note that different replicas can enter the parallel stage at different times). A cycle of the algorithm is now complete, and the replica on which the transition occurred enters the decorrelation stage, initiating a new cycle. At each cycle, the simulation time $t_{s i m}$ is incremented by the duration of the decorrelation stage, plus the sum of the MD times accumulated on all replicas during the parallel stage (blue+green). Using this algorithm, simulation time can accumulate up to $N_{r}$ times faster, in wall-clock time, than with a conventional MD simulation. Note that in the original description [31], a cycle of the algorithm begins with dephasing and ends with decorrelation. For reasons that will become clear later, we here prefer this alternative ordering. Further, the original algorithm would perform dephasing on the trajectory that just completed decorrelation. We now understand this step to be superfluous, so 
it is omitted here. Other than that last difference, the algorithm we describe here is equivalent to the original.

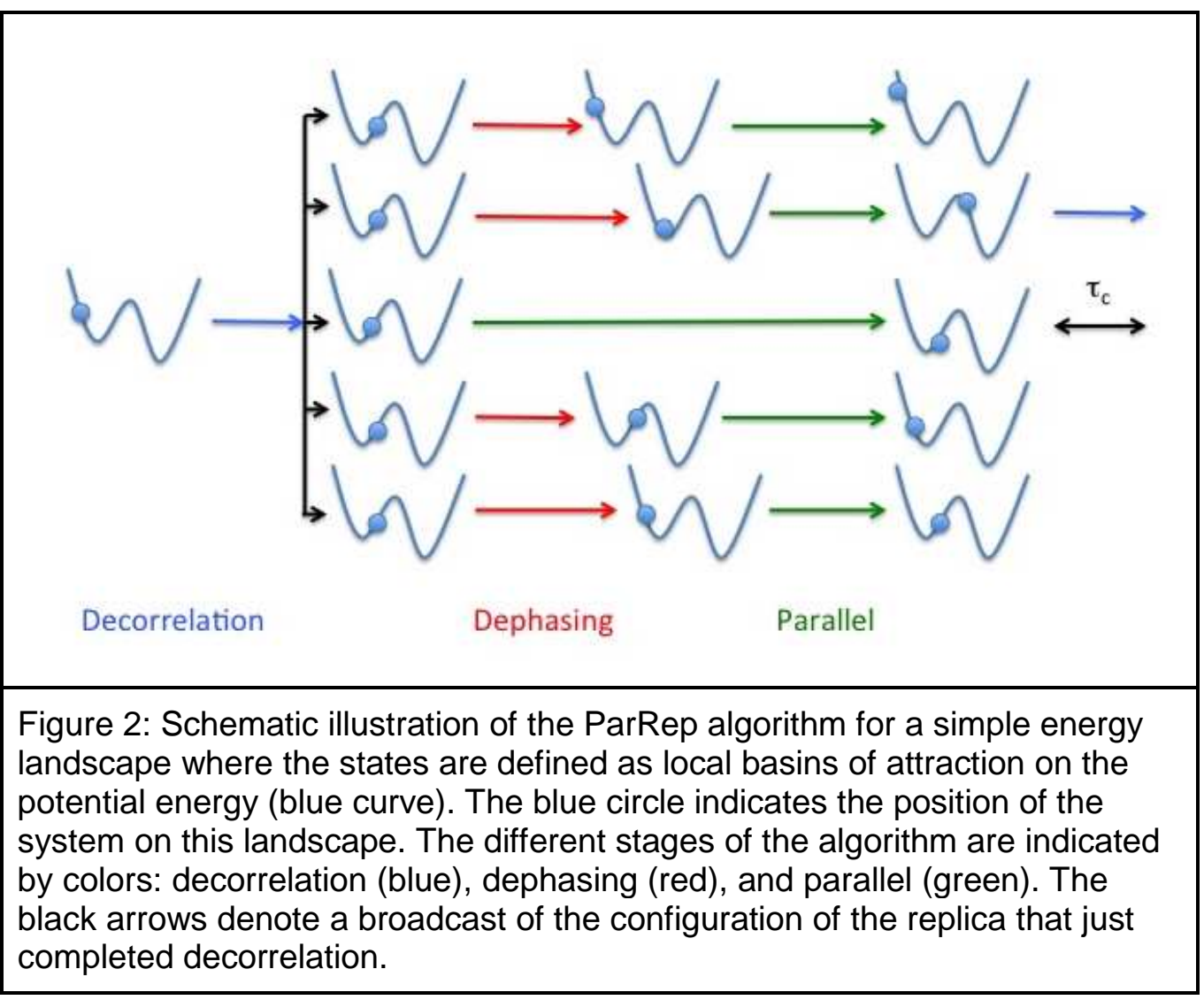

As evidenced by this description, the ParRep algorithm is extremely simple: aside from carrying out MD, the only additional requirements are that the replicas be able to detect transitions and to send messages to other replicas, for example using MPI [48]. In the common case where states are defined as basins of the potential energy surface, transition detection simply consists of periodically "quenching" configurations taken along the trajectory (using a local minimizer such as the conjugate gradient method) and comparing the result to the reference minimum. If they differ, a transition is declared. Other definitions of states require customized transition detection routines; examples of such are given below. ParRep is also free from assumptions about the possible transitions. As such, it is completely unbiased and does not require a priori assumptions on the future behavior of the system (note however that if such a priori information is available, it can be leveraged to improve the definition of states; see below). Consequently, ParRep is not designed to generate trajectories where both endpoints are specified; just like MD, ParRep solves an initial value problem.

The Markovian limit 

yields accurate results. It can however easily be shown to be exact in the important case where the state-to-state dynamics are Markovian, in a continuous-time sense. Formally, this means that the probability of the system being in state $j$ at time $t+\Delta t$ given that it was in state $i$ at time $t$ tends, as $\Delta t \rightarrow 0$, to $1-k_{i j} \Delta t$ for all $t$. Here $k_{i j}$ is the transition rate between state $i$ and $j$. This condition entails memoryless dynamics since the probability per unit time of making a transition between two states is a time- and history-independent constant. A consequence of this definition is that the distribution of escape times out of a given state, say $i$, is given by

$p_{i}(t)=k_{i} \exp \left(-k_{i} t\right)$

where $k_{i}=\sum_{j} k_{i j}$ is the total rate of escape out of state $i$. Now consider the ParRep setting with $N_{r}$ replicas that are simultaneously and independently evolved. Then, the total rate of escape, i.e., the total probability per unit time that any one replica escapes the state, is simply $N_{r} k_{i}$. The probability of a first-escape time between $t$ and $t+\Delta t$ is then

$p_{i}^{N_{r}}(t) d t=N_{r} k_{i} \exp \left(-N_{r} k_{i} t\right) d t$.

Defining the total aggregate time before the first escape as $t_{s}=N_{r} t$, the probability becomes $k_{i} \exp \left(-k_{i} t_{s}\right) d t_{s}=p_{i}\left(t_{s}\right) d t_{s}$,

i.e., the probability density of the first escape of any one of $N_{r}$ replicas after a time $t$ is identical to that of the first escape in a serial simulation after a time $t_{s}=N_{r} t$. Together with the fact that the probability that the first escape leads to state $j$ is simply $k_{i j} / k_{i}$, no matter the number of replicas, this demonstrates that ParRep generates statistically exact state-to-state trajectories if the dynamics are Markovian. This is an important result, as Markovian dynamics are the natural limit of (very) rare event dynamics. Note that the derivation relies on the statistical independence of the different replicas.

\section{Strict separation of timescales}

The decorrelation and dephasing stages in fact allow ParRep to remain exact for an even more general class of systems. Indeed, these stages would serve no purpose for truly memoryless state-to-state dynamics. They were introduced in the original formulation [31] based on the concept of separation of timescales that underpins the definition of transition rates in the chemical physics literature [11]. Such a separation implies that loss of memory in a sufficiently deep state, while not instantaneous, often occurs on a timescale that is short compared to a typical escape time. As will be discussed in more detail below, this assumption is very often justified. For example, relaxation within a harmonic well typically occurs on vibrational timescales, while transitions require exponentially longer times if barriers impede them. For the same reasons, correlated recrossings of a dividing surface (e.g., multiple jumps, bounce-backs, 
or Kramers recrossings) typically do occur only at short times. At longer times, the state-to-state dynamics can, to a very good approximation, be described in terms of rates. Under the simplifying assumption that the dynamics becomes exactly Markovian once the time spent in a state exceeds $\tau_{c}$ (what we term here a strict separation of timescales), one can show that ParRep still generates exact state-to-state dynamics. This is so because the complex nonMarkovian behavior is captured by direct dynamics during the decorrelation phase. At the end of that phase, the system becomes Markovian and the demonstration given above applies. This is a first indication that the applicability of ParRep extends beyond the realm of simple Markovian systems.

\section{Extension to the general case using Quasi-Stationary Distributions}

While it is certainly reassuring to know ParRep is exact for dynamics that become strictly Markovian after some time, the fact that atomistic state-to-state dynamics are, formally speaking, not perfectly Markovian at any finite time might be cause for concern. For example, one might worry that slight departures from Markovianity could lead to errors that magnify uncontrollably as a result of the ParRep procedure. This could become a problem when dealing with weakly metastable states (where intra-state equilibration and inter-state transitions are not well separated in time) or when using a very large number of processors. Over the last few years, the understanding of the formal underpinnings of ParRep has dramatically improved based on a very simple realization: in the context of ParRep, Markovianity should not be seen as an absolute property of the dynamics, but as a condition that is inexorably approached on sufficiently long timescales. A consequence of this understanding is that ParRep can be shown to be able to deliver arbitrary accuracy, even for dynamics that are not strictly Markovian at any finite time. The rate at which the system approaches Markovianity is then the key parameter that controls the efficiency of ParRep. In the following, we demonstrate this unique property by mirroring the derivation first presented in Ref. [47]. We err on the side of simplicity and readability, at the expense of mathematical rigor. Readers interested in the mathematical subtleties of the proof are referred to the original manuscript. To better convey the key aspects in the simplest manner, we first consider the problem using the formalism of continuous-time Markov chains; we then proceed to generalize the results to the case of overdamped dynamics on a continuous potential, as in Ref. [47], and discuss the reinterpretation of ParRep in light of these results.

Consider a continuous-time Markov chain on a finite state space, i.e., a KMC model. The chain is fully specified by its generator matrix $Q$, defined such that $Q_{i \neq j}=k_{i j}$ and $Q_{i i}=-k_{i}$, where the transition rates $k_{i j}, k_{i}$ are the transition rates from state $i$ to state $j$, and the total transition rate out of state $i$, respectively. If no direct transition pathway exists between two states, the corresponding rate is set to zero. We assume the chain to be reversible, i.e., to obey detailed balance. Now define a meta- or super-state $\alpha$ as an arbitrary connected subset of states. Denote the complement of $\alpha$ by $\gamma$. We are interested in the statistics of the first escape to $\gamma$ of a trajectory that begins in $\alpha$. The natural setting to study this problem is that of absorbing Markov 
chains [49]. The generator of the absorbing chain $Q_{\alpha}$ is the submatrix of $Q$ containing only the elements $i, j$ where both $i$ and $j$ are in $\alpha$. Note however that the diagonal elements of $Q_{\alpha}$ contain the total rate of escape out of each of the states in $\alpha$, including transitions to states in $\gamma$; i.e., $Q_{\alpha}$ does not constrain trajectories to stay in $\alpha$ but prohibits trajectories that left $\alpha$ from re-entering. Therefore, the rows of $Q_{\alpha}$ do not sum to zero, as is the case for ordinary (non-absorbing) Markov chains, which precludes the existence of a stationary distribution. The equation of motion for the probability $P$ of being in the different sub-states of $\alpha$ at time $t$ is given by

$\partial_{t} P=P Q_{\alpha}$,

so that, formally,

$P(t)=P_{0} \exp \left[Q_{\alpha} t\right]$

If $Q$ obeys detailed balance, then $Z^{1 / 2} Q_{\alpha} Z^{-1 / 2}$ is a symmetric matrix which admits a spectral decomposition $Z^{1 / 2} Q_{\alpha} Z^{-1 / 2}=\sum_{j=1}^{N} \lambda_{j} x_{j} x_{j}{ }^{T}$, in term of its eigenvalues $-\lambda_{j}$ (sorted in ascending order) and eigenvectors $x_{j}$. Here, $Z$ is a matrix whose diagonal contains the equilibrium distribution in each state on the original chain $Q$. It then follows that

$P(t)=\sum_{j=1}^{N} \exp \left[-\lambda_{j} t\right]\left(P(0)^{T} v_{j}\right) u_{j}^{T}$,

with $u_{j}=Z^{1 / 2} x_{j}$ and $v_{j}=Z^{-1 / 2} x_{j}$. Because the chain is absorbing we have $\lambda_{1}>0$, which implies that the survival probability $\sum_{k} P_{k}(t)$ (the probability of still being somewhere in $\alpha$ at time $t$ ) is not constant but decays to zero as $t \rightarrow \infty$. This decay corresponds to trajectories that left $\alpha$ to enter $\gamma$, i.e., $P_{k}(t)$ is the probability that a trajectory is in state $k$ at time $t$ without having left $\alpha$ at any earlier point in time. At short times, the probability distribution contains a contribution from every one of the $N$ relaxation modes, each weighted by a factor that depends exponentially on their respective $\lambda$. The escape dynamics are obviously not Markovian at this stage as the survival probability is not given by a single exponential. For $t \gg 1 /\left(\lambda_{2}-\lambda_{1}\right)$, however, only one of these modes contributes significantly and the distribution becomes

$P(t) \simeq \exp \left[-\lambda_{1} t\right]\left(P(0)^{T} v_{1}\right) u_{1}^{T}$,

i.e., at long times, the distribution is almost proportional to $u_{1} \cdot u_{1}$ is referred to as the quasistationary distribution (QSD), owing to the fact that it is the unique distribution that is left invariant by the dynamics, conditional on the chain never having left $\alpha$. Again, because $\lambda_{1}>0$, this distribution is not stationary because, under $Q_{\alpha}$, the survival probability constantly decays -hence the name quasi-stationary. This confers on the QSD a crucial property: from the QSD, first escape from $\alpha$ is a Markovian process. This can be seen from the fact that the survival probability starting from the QSD decays exponentially and that the probability of escaping $\alpha$ from state $i$ if the escape occurs at time $t$, 
$P_{i}(t) k_{i \gamma} / \sum_{j} P_{j}(t) k_{j \gamma}=u_{1, i} k_{i \gamma} / \sum_{j} u_{1, j} k_{j \gamma}$

is time-independent. Combined with the fact that the dynamics, conditional on not yet having escaped, converges to the QSD from any initial condition, one obtains the following result: conditional on not having escaped at time $t \gg 1 /\left(\lambda_{2}-\lambda_{1}\right)$, the first escape out of any set $\alpha$ is almost Markovian. Thus, Markovianity should not be seen as absolute, but as a property that progressively develops with time. Note that, in this context, Markovian behavior is obtained in the limit $\lambda_{2} \rightarrow \infty$, i.e., it corresponds to a case where the QSD is instantaneously established.

These results can be illustrated using a simple 1D model potential composed of a number of basins, shown in Figure 3. We discretize the continuous state space by assigning one state to each basin and define transition rates as $k_{i j}=\exp \left[-\beta\left(E_{i j}{ }^{*}-E_{i}\right)\right]$ with $E^{*}{ }_{i j}$ the energy of the saddle point (maximum) between basins $i$ and $j, E_{i}$ the energy of the minimum in basin $i$, and $\beta=1 / k_{B} T$. This simple choice leads to a reversible chain. Defining $\alpha$ as containing the nine central basins (between $X=0.5$ and $X=1.5$ ), the left panel of Figure 4 shows how the QSD differs from the equilibrium Boltzmann distribution (normalized to unity over $\alpha$ ). The main difference is that the QSD is depleted close to the edge of the super-state and enriched towards the center. This results from the conditioning on never having left the state. Indeed, a (potentially significant) fraction of the trajectories found in an edge state on the original chain would have visited $\gamma$ in the recent past. The contribution from these trajectories is excluded in the QSD, hence the depletion. Note that the high temperature $(\beta=1)$ used in this example magnifies the difference between the two distributions; it is much smaller in practice for strongly metastable states. The corresponding survival probability is plotted in the right panel for $P_{0}(X)=$ const. The probability is non-exponential at short time, but then decays exponentially. The green line corresponds to the Markovian limit where $P_{0}(X)=u_{1}(X)$. As expected, the survival probability decays with exponent $\lambda_{1}$ at sufficiently long times. The difference between the two curves is a reflection of the finite amplitude of the non-QSD modes when starting from the uniform distribution. It is hence a measure of the inefficiency of the dephasing stage and indicates that rejections would occur during dephasing. 

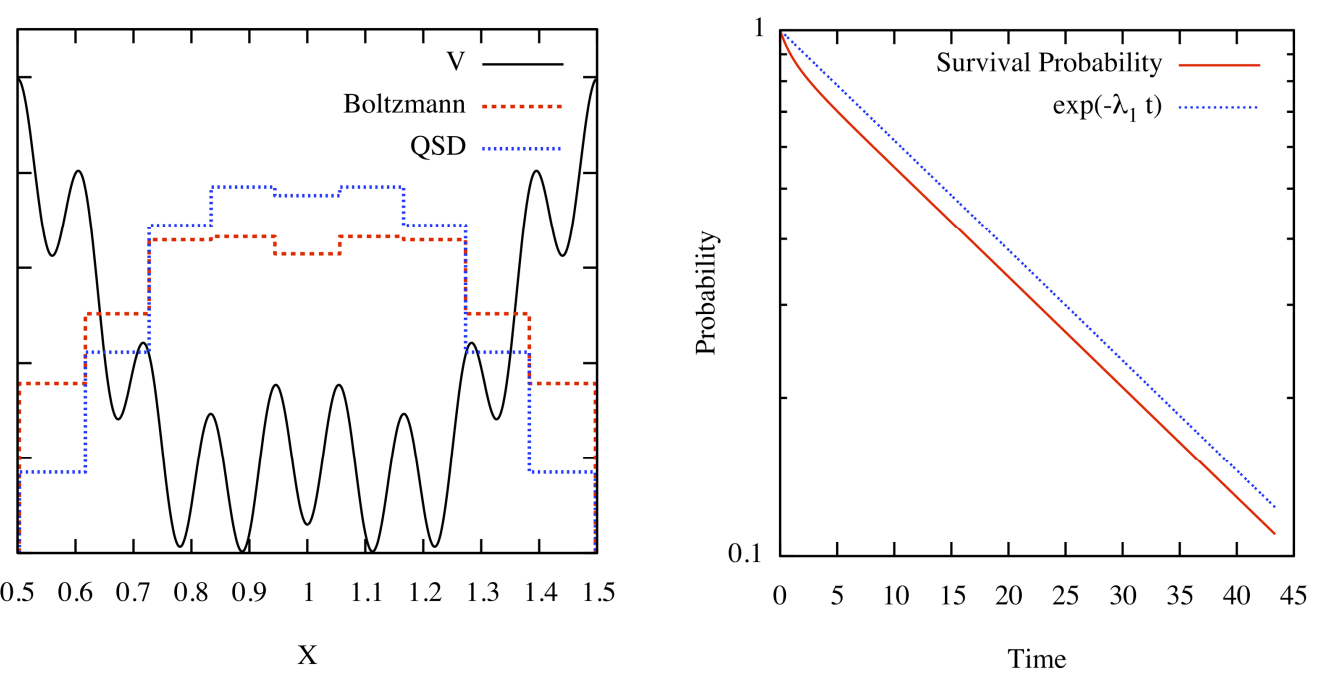

Figure 3: Model potential used in the numerical experiments. See text for details.

Figure 4: Numerical results for a discrete Markov chain inferred from the model potential for a state containing nine basins at $\beta=1$. Left: Boltzmann distribution (red dashed line) and QSD (blue dotted line). The continuous potential is shown in black. The state extends from $X=0.5$ to 1.5. Right: Survival probability as a function of time for a uniform initial distribution (red). The dashed blue line shows the expected long time limit. 
These results are not limited to Markov chains but can be generalized to arbitrary states defined on continuous state-spaces [47]. Consider a system evolving on a potential $V(X)$ at inverse temperature $\beta$ through overdamped Langevin dynamics. In units where the friction coefficient and the masses are unity, the equations of motion are given by

$d X_{t}=-\nabla V\left(X_{t}\right) d t+\sqrt{2 \beta^{-1}} d W_{t}$,

where $d W_{t}$ is a delta-correlated stationary Gaussian process with zero-mean and unit variance. Define a state $\alpha$ as the region of configuration space enclosed in a connected (hyper-)surface $\Omega_{\alpha}$. We are again interested in the statistics of first escape of trajectories that start in $\alpha$. Consider $P(X, t)=P\left(X, t \mid P_{0}, 0\right)$ as the probability density function for the system to be at $X$ at time $t$ given that it was distributed according to $P_{0}$ at time 0 and that it never left $\alpha . P(X, t)$ then obeys the following Smoluchowski equation:

$\partial_{t} P=L P$

in $\alpha$ with boundary condition $P=0$ on $\Omega_{\alpha}$, where $L=-\nabla \cdot(\nabla V \cdot)+\beta^{-1} \nabla^{2}$. Using a spectral decomposition of $L$ in terms of its eigenvalues $-\lambda_{i}$, again sorted in increasing order, and eigenfunctions $u_{i}(X)$, one obtains

$P(X, t)=\sum_{i} c_{i}^{0} \exp \left(-\lambda_{i} t\right) u_{i}(X)$

where the $c_{i}{ }^{0}$ are constants such that $P(X, 0)=P_{0}(X)$, i.e. $c_{i}{ }^{0}=\int P_{0}(X) u_{i}(X) d \mu^{-1}(X)$, where $\mu(X)$ is the invariant measure of the original dynamics. For $t \gg 1 /\left(\lambda_{2}-\lambda_{1}\right)$, we get

$P(X, t) \simeq c_{1}^{0} \exp \left(-\lambda_{1} t\right) u_{1}(X)$.

This expression is equivalent to that derived above for Markov chains and the QSD $u_{1}$ can be shown to have the same properties, namely that the first escape out of $\alpha$ is Markovian for $P_{0}(X)=u_{1}$ and that $P\left(X, t \gg 1 /\left(\lambda_{2}-\lambda_{1}\right)\right) \simeq c_{1}{ }^{0} \exp \left(-\lambda_{1} t\right) u_{1}(X)$ for all $P_{0}(X)$ and for every $\alpha$ [47]. In this case also, Markovianity should not be seen as absolute, but as a property that progressively develops with time. These results can be illustrated using the simple model introduced earlier. As shown in Figure 5, the results are qualitatively similar to the discrete Markov Chain case. The QSD again shows a depletion towards the edges, and the tail of the survival probability also decays as $\exp \left[-\lambda_{1} t\right]$ at sufficiently long times. 

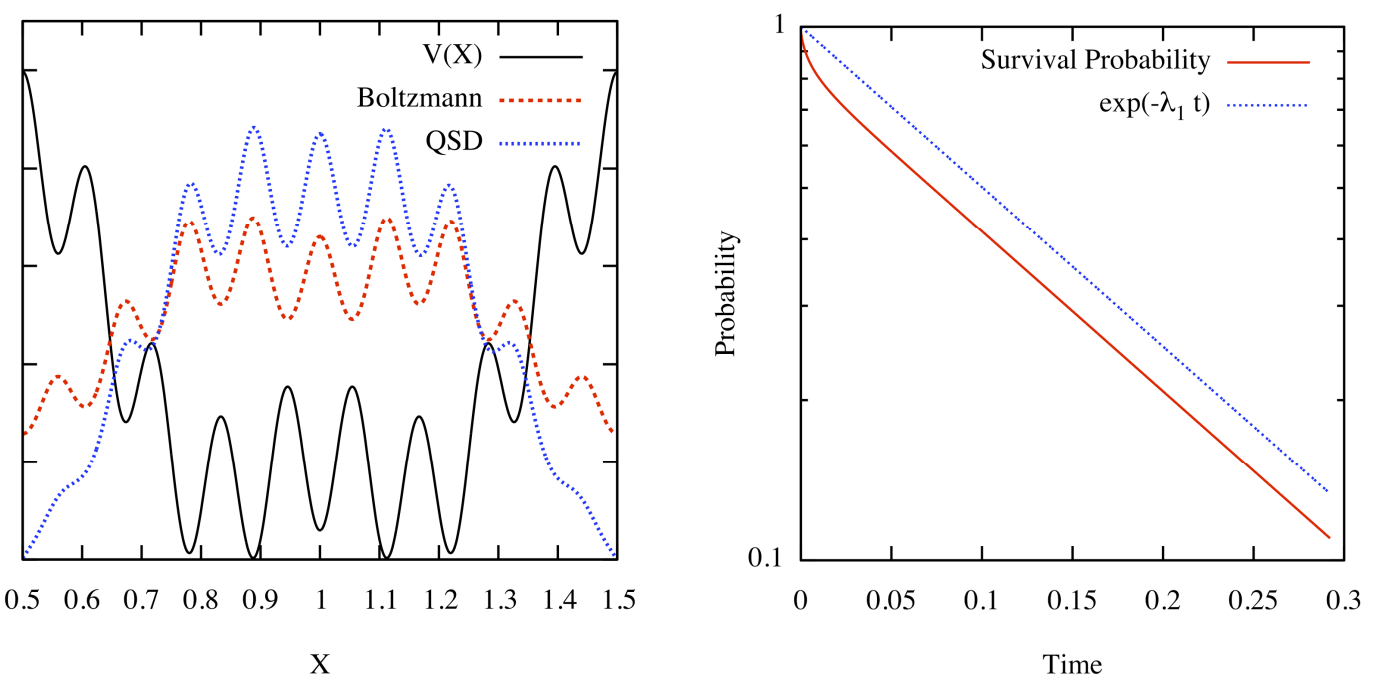

Figure 5: Numerical results for overdamped Langevin dynamics using the model potential for a state of unit width centered at $\mathrm{X}=1$ at $\beta=1$. Left: Boltzmann distribution (red dashed line) and QSD (blue dotted line). The continuous potential is shown in black. The state extends from $X=0.5$ to 1.5. Right: Survival probability as a function of time (red) for a uniform initial distribution. The dashed blue line shows the expected long time limit.

\section{Errors in ParRep}

These results allow for a reinterpretation of the ParRep algorithm and for a better understanding of the errors. Consider a KMC simulation on a discrete space or an MD trajectory. If the trajectory spends more than $\tau_{c}>\left(\lambda_{2}{ }^{\alpha}-\lambda_{1}{ }^{\alpha}\right)^{-1}$ without leaving a certain state $\alpha$, then its end point will be almost distributed according to the QSD $u_{1}{ }^{\alpha}$ for that state (decorrelation). At this point, introduce $N_{r}-1$ other replicas in $\alpha$ and run until they remain for at least $\tau_{c}$ within $\alpha$, retrying as necessary (dephasing). At the end of this stage, the endpoints of these trajectories will also be independent samples from a distribution that approaches $u_{1}{ }^{\alpha}$. Note that statistical independence requires each replica to dephase on its own, i.e., dephased points cannot be shared between replicas. Run on all replicas until the first escape and increment the simulation clock using the time accumulated by all replicas during that last stage together with the time accumulated by the original replica while decorrelating. According to the previous discussion, this first escape of any of the replicas will be almost Markovian. This procedure is a direct translation of the ParRep algorithm sketched above into the language of QSDs. In the original formulation, decorrelation was meant to allow for correlated post-transition recrossings to occur, while dephasing was introduced to achieve statistical independence between replicas. In this new understanding, decorrelation and dephasing are formally equivalent: their purpose is to enforce that the QSD is reached before the parallel stage begins. The advantage of the new formal framework is that the effect of the deviations from the ideal Markovian behavior can now be quantified: it has been shown that the expected error of arbitrary functions of the escape 
points (the hitting points of escaping trajectories on $\left.\Omega_{\alpha}\right)$ and times is of order $\exp \left[-\left(\lambda_{2}{ }^{\alpha}-\right.\right.$ $\left.\lambda_{1}{ }^{\alpha}\right) \tau_{c}$ ] [47]. Thus, ParRep can be made arbitrarily accurate for any definition of states, simply by adjusting $\tau_{c}$. This result might seem surprising, as one could have expected finite errors stemming from imperfect Markovianity. But, as argued above, Markovianity is not absolute: at long enough times, many systems (e.g., those evolving through Langevin dynamics) will exhibit Markovian first-escape dynamics. The key to the accuracy in ParRep is to use the decorrelating trajectory to capture the complex short time behavior, when many modes contribute to the evolution, and to trigger the parallel stage only when the QSD is approached, i.e., when the decorrelating trajectory has spent long enough in each state. The dephasing/decorrelation procedure is now interpreted as a rejection-based strategy to generate independent samples from the QSD, and not simply as a tool to achieve statistical independence. In that context, $\tau_{c}$ measures the time needed for the weight of the QSD in the total probability distribution to dominate over that of other modes. The crucial importance of properly defining states and of estimating $\tau_{c}$ is addressed below.

\section{Efficiency of ParRep}

Of course, as an acceleration method, the usefulness of ParRep is judged by its ability to extend the simulation timescales. Remembering that dephasing does not advance the simulation clock, we see that ParRep will be simultaneously accurate and efficient as long as $\left(\lambda_{2}-\lambda_{1}\right)^{-1}<\tau_{c}<$ $\lambda_{1}^{-1} / N_{r}$, taking $\lambda_{1}^{-1}$ as an approximation of the (single replica) escape rate, and hence $\lambda_{1}{ }^{-1} / N_{r}$ as the duration of the parallel stage. This expression shows that a crucial requirement for ParRep is the availability of states with large spectral gaps $\left(\lambda_{2}-\lambda_{1}\right)$, insuring that the correlation time will be well separated from the first escape time. In the worst case, when the spectral gap is very small, a transition will almost always occur before $\tau_{c}$ and the algorithm will never exit the decorrelation stage. In this limit, ParRep naturally reduces to standard MD, albeit one with a poor parallel efficiency of order $1 / N_{r}$. If however, the spectral gap is large, the parallel efficiency (the rate at which the simulation time advances in ParRep relative to the rate at which the total time accumulates for $N_{r}$ independant conventional MD simulations) approaches 1, leading to speedups of order $N_{r}$ over standard MD. In between these two limits, the parallel efficiency will scale roughly as

$\frac{1}{\tau_{c} \lambda_{1} N_{r}+1}$ 

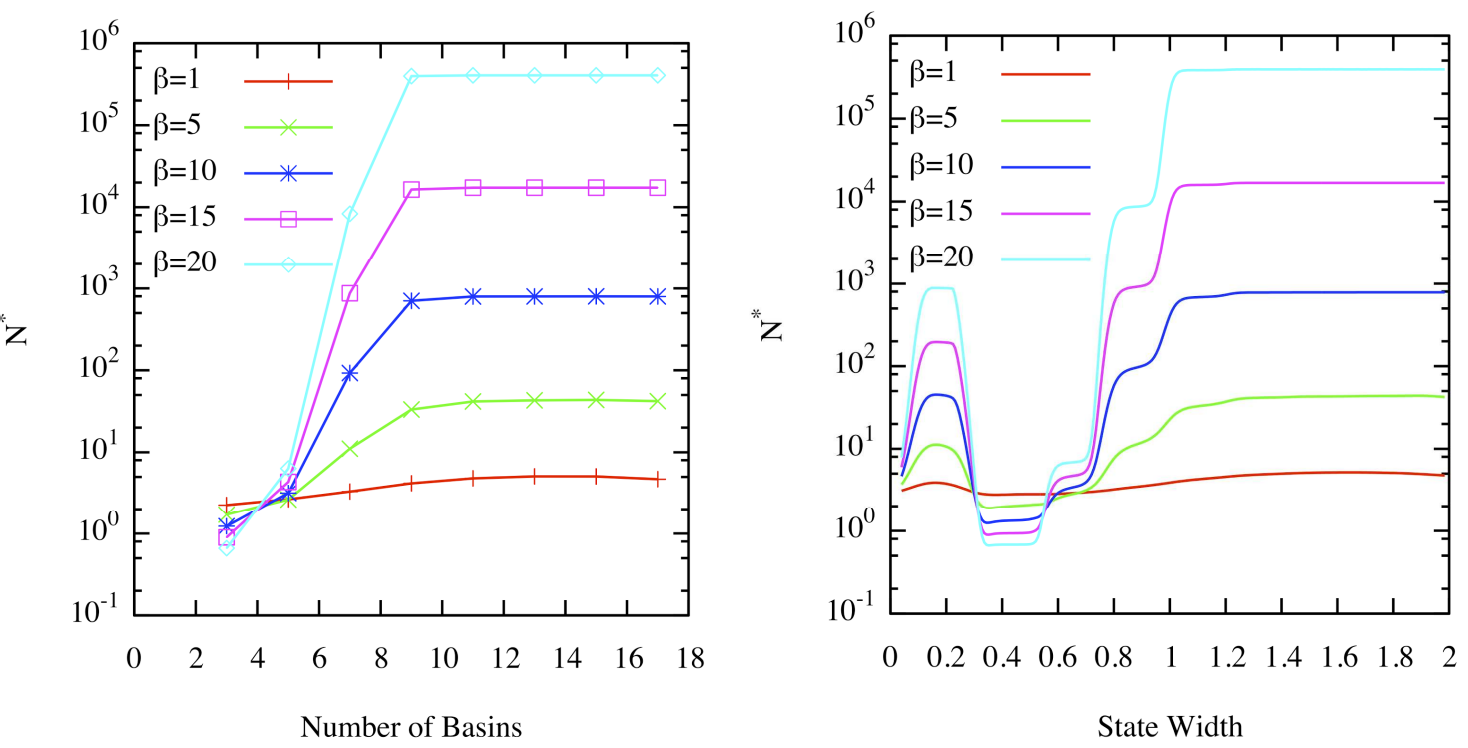

Figure 6: Scalability metric $N^{*}=\left(\lambda_{2}-\lambda_{1}\right) / \lambda_{1}$ for different states defined symmetrically around $X=1$ at different temperatures for the potential shown in Fig. 3. Left: Discrete Markov Chain results; Right: Overdamped Langevin results.

\section{States and Correlation Times}

As a general rule, $u_{1}$ is the only mode that can be interpreted as a probability distribution (as it does not change sign); as discussed above, $u_{1}$ is the QSD and $\lambda_{1}$ is the corresponding escape rate. The other modes roughly correspond to transfer of probability within the state, from regions where the modes are negative to regions where they are positive (or vice-versa). To first order, these higher modes control the relaxation of the shape of the probability distribution. When using energy basins as states, the intra-state relaxation rates are controlled by vibrational frequencies and the friction coefficient (the strength of the coupling to the thermostat) [50]. For typical solid state systems of moderate size ( 1000 atoms), one then typically has $\tau_{c} \simeq 1 p s . \lambda_{1}$ corresponds to the escape rate from the state and hence is of order $v_{c} \exp \left[-\beta \Delta E_{\min }\right]$ with $v_{c}$ a typical vibrational frequency and $\Delta E_{\min }$ the smallest energy barrier that has to be overcome to leave the state. For $\Delta E_{\min } \gg \beta^{-1}$, we therefore expect a large spectral gap and ParRep should be very efficient, insofar as $N_{r}$ is such that $\tau_{c} \leq \lambda_{1}{ }^{-1} / N_{r}$. As a rule of thumb, the maximum number of replicas one can use while maintaining reasonable efficiency can be approximated as the number of replicas for which the correlation time $\left[\sim\left(\lambda_{2}-\lambda_{1}\right)^{-1}\right]$ is equal to the time before some replica sees the first escape from the state $\left(\lambda_{1}{ }^{-1} / N_{r}\right)$, i.e.,

$N^{*}=\left(\lambda_{2}-\lambda_{1}\right) / \lambda_{1}$. 
Unfortunately, $N^{*}$ can be quite low if $\Delta E_{\min }$ happens to be small $\left(\lambda_{1}\right.$ large); this is the so-called low-barrier problem. If, however, these low barriers only lead to motion between a limited number of basins, one can define a (super-)state such that all low barriers are contained within it, leaving comparatively large barriers for escape to the outside. Since super-states contain internal barriers, $u_{2}$ now corresponds to moving probability across the largest intra-state barriers, a process that proceeds at a rate $\lambda_{2}$, which again will be roughly exponentially small as a function of the height of the said internal barriers. While such a super-state will have a lower value of $\lambda_{2}$ compared to a single basin, it might also have a (much) lower value of $\lambda_{1}$ because the inter-state transition rate can be made smaller by lumping low barriers within the superstate, leaving only high barriers leading to the outside. Super-states can hence have a larger $N^{*}$ than any individual basins, restoring the efficiency of ParRep.

This is illustrated in Fig. 6, where $N^{*}$ is shown for increasingly large states centered around $X=1$. In the discrete case, lumping does not initially provide any benefit, as intra-state barriers are larger than inter-state ones. This leads to values of $N^{*}$ close to unity, indicating that ParRep offers no advantage over direct serial simulation. $N^{*}$ even dips below unity as the temperature decreases ( $\beta$ increases), because equilibration within the state is increasingly difficult due to the high intra-state barriers. $N^{*}$ then starts increasing with the size of the state because of the progressively higher inter-state barriers. The temperature dependence now reverses and efficiency increases dramatically with decreasing temperature. This is a consequence of the inter-state transitions slowing down faster than intra-state ones, leading to an increase in the spectral gap. The results also show that, once the state encompasses the highest ridge top in a local region of the landscape, the precise location of the boundary does not significantly affect performance, as long as it does not start encompassing high barriers further out. A very similar behavior is observed in the overdamped Langevin case, except for a peaked feature around a width of 0.2. These states, roughly equivalent to the conventional single basin definition, enable good performance. As shown in the left panel of Fig. 7, the higher order relaxation modes $u_{2}$ and $u_{3}$ correspond to transfer of probability between the left and right parts, or from the middle to the sides, respectively. As no barrier impedes this transfer of probability, they are associated with large values of $\lambda$. However, extending their range too far into the neighboring basins leads to a rapid decrease in $N^{*}$. This is caused by the introduction of slow relaxation modes which can be seen in the middle panel of Fig. 7 . Now $u_{2}$ corresponds to transferring probability density between the outer basins, the rate of which is limited by high barriers, and $u_{3}$ corresponds to relaxation between the central basin and the outer ones, again a slow process. Since the intrastate barriers that control $\lambda_{2}$ are of the same order as the inter-state barriers that control $\lambda_{1}$, the spectral gap, and hence $N^{*}$, is small. Note that this regime is absent from the discrete case, as a single state is assumed to have an infinite spectral gap in the Markovian approximation, and hence is not plotted. Comparison with the Langevin results shows the Markovian assumption to be unwarranted when $\beta^{-1}$ is of the same order as the inter-basin barriers. As the size of the states increases further, $N^{*}$ is seen to dramatically increase again, as in the discrete case. As shown on the right panel of Fig. 7, this now corresponds to cases where overcoming the highest barriers is not the rate limiting step for any of the internal relaxation modes, as the regions where $u_{2}$ and $u_{3}$ are large are concentrated inside of the state, not on both sides of the largest 


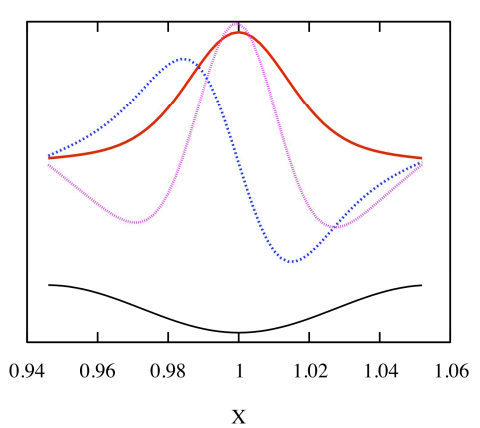

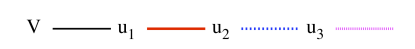

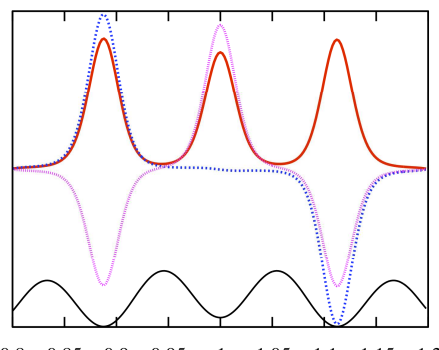

$\begin{array}{lllllllll}0.8 & 0.85 & 0.9 & 0.95 & 1 & 1.05 & 1.1 & 1.15 & 1.2\end{array}$

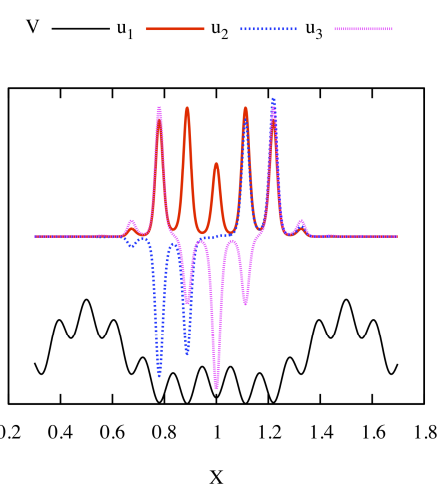

Figure 7: The first three eigenfunctions of the generator of the overdamped Langevin dynamics for different states. Left: width $=0.1\left(\lambda_{1}=14.06, \lambda_{2}=445.49, \lambda_{3}=848.36\right)$; Middle: width $=0.4\left(\lambda_{1}=2.45, \lambda_{2}=5.68, \lambda_{3}=10.43\right)$; Right: width $=1.4\left(\lambda_{1}=1.09 \times 10^{-3}, \lambda_{2}=\right.$ $\left.0.85, \lambda_{3}=5.13\right)$.

In practice, the challenge in exploiting this flexibility in state definition is twofold: first define states such that the relaxation times are (much) shorter than the escape times, and, second, estimate $\tau_{c}$. Different methods have been proposed to address the first issue, especially in the context of KMC simulation [51], but also for the creation of Markov models for bio-molecules [52]. The problem is slightly simpler here, as one only needs to find "good" definitions, not "perfect" ones, so that heuristics could be sufficient at this stage. For example, simply observing that a trajectory is trapped within the same set of states for a long time suggests that this set could be a good super-state. The second problem is more subtle. If the number of individual basins within each state is sufficiently small and if the basin-to-basin dynamics can be considered Markovian to a good approximation, then the formalism of absorbing Markov Chains discussed above can be used to estimate the spectral gap. The procedure would involve the approximation of the generator $Q_{\alpha}$ from simulation data and the estimation of its spectral gap. This can be done rigorously, including the quantification of the errors due to limited statistics $[53,54]$. If, however, Markovian dynamics cannot be assumed at the basin-to-basin level -- for example, if $\Delta E_{\min } \simeq \beta^{-1}$, and/or if the number of basins is overwhelming -- then estimating the spectral gap is largely an open problem if one cannot rely on specific physical insights. Indeed, the problem would then be akin to forming and diagonalizing a Fokker-Planck operator in a very high dimensional space, a prohibitively expensive task using brute force approaches. However, a more scalable strategy that aims at directly estimating the extremal eigenvalues has recently been introduced [55]. It has also been proposed that certain statistics could be used to test the stationarity of well-chosen observables in order to estimate the correlation time [56]. It is important to remember that, in the context of ParRep, we do not need to precisely determine the spectral gap, but merely to bound it so as to obtain a safe estimate of $\tau_{c}$. Defining states in the absence of an underlying discrete Markovian dynamics is also potentially challenging, but again, 
one has considerable license in doing so, as they could be defined in a properly chosen subspace of the full configuration space, e.g., in terms of the dihedral angles of the backbone of a protein. Physical intuition can be expected to play an important role in defining states for novel classes of systems.

As physical intuition sometimes falls short, automated techniques to define states or to detect transitions would also be extremely desirable. Strategies have been proposed in order to achieve this goal. For example, Meerbach et al. have proposed a change point detection scheme to automatically detect transitions under the assumption that the dynamics can locally be approximated by a harmonic model [57]. Interestingly, the change point detection is not based on the trajectory itself, but on the parameters of the approximate model that locally describes the dynamics. In this approach, state boundaries are not explicitly constructed; instead one aims at detecting changes in the behavior of the system that could be attributed to a transition. It is our belief that methods such as these will play an important role in the future of ParRep, but, at present, practical hurdles still have to be overcome before ParRep can live up to its full potential.

This discussion should make clear why the single-basin state definition is by far the most convenient in practice: one can easily estimate a single value of $\tau_{c}$ that is valid for all the states, and transitions can be simply and reliably detected. Conversely, if the states are not defined based on a separation of timescale that can be quantified using physical arguments, $\tau_{c}$ has to be determined for every state, a procedure that can entail algorithmic complexity and a significant computational investment. Therefore, it is advisable to first assess whether the conventional definition allows for sufficient acceleration before considering more complex alternatives.

\section{ParRep for Driven Systems}

Up to now, we have considered static systems, where the potential or the boundary conditions do not change with time. However, in materials science applications, it is often desirable to simulate the effect of "loading", for example, imposing a given strain rate by changing the boundary conditions in time. Can ParRep be used to accelerate such a system? It is straightforward to show that it can [58]. We now place ourselves in the setting of timeinhomogeneous Markov chains, i.e., Markov chains where the transition rates are time dependent, and assume that the time dependence of the rates only comes through the instantaneous value of the driving parameter $B(t)$ (e.g., the position of a clamp that is varied with time). This is equivalent to a quasi-static limit where the instantaneous rates are dependent on the instantaneous value of the driven parameter but independent of the driving schedule. In this case, the probability density of escape time out of the state becomes

$p_{B}(t)=[B(t)] \exp \left[-\int_{0}^{t} k\left[B\left(t^{\prime}\right)\right] d t^{\prime}\right]=k[B(t)] \exp \left[-F_{B}(t)\right]$. 
Now consider a driving schedule $B^{\prime}(t)=B\left(N_{r} t\right)$ that drives the system $N_{r}$ times faster. Our assumptions imply that $k\left[B\left(N_{r} t\right)\right]=k\left[B^{\prime}(t)\right]$. It is then simple to show that $F_{B}(t)=F_{B}\left(N_{r} t\right) / N_{r}$. From this point, the derivation is identical to the Markovian case presented above, and we get $p^{N_{r}{ }_{B}}(t) d t=p_{B}\left(t_{s}\right) d t_{s}$, i.e., the probability of a transition per unit time under schedule $B$ after a time $t_{s}=N_{r} t$ is equal to that of the first of $N_{r}$ replicas under schedule $B^{\prime}$. Therefore, one can apply the conventional ParRep algorithm to such a scenario, except that the drive now proceeds $N_{r}$ times faster on each processor. Note that one must now proceed with the first transition to occur in MD time, which requires that all replicas run at least up to the putative first transition time before it can be certified as the true first transition. In contrast, in the undriven case, no such restriction applies and one can proceed with the first transition that occurred in wall-clock time.

It is important to emphasize that this derivation only holds for driving schedules $B$ and $B^{\prime}$ that are both quasistatic. If one wishes to use a large number of processors, this assumption may break down for $B^{\prime}$ even if it applies to $B$. In this case, it would be advisable to load in discrete steps instead and to re-dephase after each load increment. This way, one can ensure the quasistatic nature of the loading, albeit at the cost of additional dephasing steps.

\section{ParRep in Practice}

As discussed above, ParRep is an extremely flexible simulation methodology. Since its inception in 1998, it has been used to simulate a wide range of material systems, including the diffusion of $\mathrm{H}_{2}$ in crystalline $\mathrm{C}_{60}$ [59], the pyrolysis of hexadecane [60], the transformation of voids into stacking fault tetrahedra in FCC metals [23], the stretching of carbon nanotubes [58], grain boundary sliding in $\mathrm{Cu}$ [61], friction-force microscopy [62,63], the diffusion of Li through a polymer matrix [64], the fracture process in metals [65] and the folding dynamics of small proteins [66]. The goal here is not to provide an extensive review of all of these applications but to illustrate some of the key points addressed above and provide context and inspiration to the reader wishing to use ParRep to study novel classes of materials, especially with respect to the definition of states.

\section{States as energy basins}

The traditional and most straightforward definition of states in ParRep is to equate states and potential energy basins. This leads to a very simple transition detection procedure, and allows for the a priori estimation of $\tau_{c}$, for example using analytical solutions for the Fokker-Planck equation for harmonic basins. It has therefore been the definition of choice in many applications $[23,58,61,62,63,65]$. This definition is warranted when the barriers between states are large compared to the thermal energy, making the transition rate sufficiently low. Fortunately, this is often, though not always, the case for hard materials around room temperature, especially for crystalline solids with limited numbers of defects. 
For example, ParRep has been used to model friction force microscopy (FFM) experiments. In FFM, a cantilever that terminates in a very sharp tip is brought in contact with the surface of interest. The cantilever is then dragged, usually at constant speed, across the surface. Because of the adhesion between the tip and the surface, elastic loading of the cantilever usually develops as it moves along. The resulting deformation trace, obtained by optically measuring the deflection of the cantilever, can be used to infer the frictional behavior of the contact, i.e., to measure the energy loss as the contact travels along the surface. The main difficulty in using atomistics to simulate such an experiment is that experimental driving rates are extremely low, typically in the $1-100 \mathrm{~nm} / \mathrm{s}$ range. While it might not be essential to exactly match the experimental velocity in order to extract the relevant physics, it was shown that typical MD speeds might in fact probe a completely different frictional regime that is irrelevant to the interpretation of the experiments [67]; achieving long timescales is therefore paramount for qualitatively correct conclusions to be reached. The driven version of ParRep has been shown to be an effective tool to approach the problem [68]. Indeed, when studying friction on clean surfaces, the contact usually evolves in a stick-slip fashion, i.e., the system alternates between phases of elastic loading, where the contact is basically pinned in a potential energy basin created by its interaction with the surface, leading to a continuous elastic loading of the cantilever, and very fast slips, where the contact quickly jumps to a new basin, releasing some load. The basin-based state definition is ideal to capture these slips, as well as other conformational changes on the contact. In Ref. [67], this approach has been demonstrated using an Au surface scanned with a Pt coated tip. By using around 500 replicas to decrease the driving speeds from $10^{8} \mathrm{~nm} / \mathrm{s}$ to about $10^{6} \mathrm{~nm} / \mathrm{s}$, the thermally activated regime typical of experiments was probed and good agreement was achieved with measurements for the critical force and potential shape parameters. The main source of the remaining discrepancy concerns the kinetic prefactor of the slip rate; the difference was attributed to the widely differing inertia of the atomistic (1000's of atoms) and real (100's of $\mu \mathrm{m}$ long) cantilevers, an issue whose resolution calls for spatial multiscaling techniques.

While the basin-based definition is probably not optimal in most cases, it nonetheless can lead to extremely high performance when barriers are sufficiently high. For example, ParRep was ported to the Roadrunner supercomputer at Los Alamos National Laboratory. Roadrunner was the first computer to break the peta-flops $\left(10^{15}\right.$ operations/s) barrier. This implementation was used to study the plastic deformation of silver nanowires during tensile tests, using wires similar in shape and size to those used in in-situ TEM experiments [69]. These wires were a few $\mathrm{nm}$ long, about $1 \mathrm{~nm}$ wide, and were oriented along the [110] direction. The evolution of the wires in a typical simulation is illustrated in Fig. 8. During the initial phase of the simulation, the wires stretch elastically. The first deformation mechanism to activate consists of slip along (111) planes, leaving stacking faults behind. At low strain rates, these stacking faults are transient, as they can thermally annihilate through the reverse process. At high strain rates, in contrast, these are basically frozen in place. As strain increases, more and more of these stacking faults are present in the wire, until complete saturation is achieved when six of them coexist in a zigzag arrangement. Note that, as slip is the first process to become spontaneous due to a vanishing barrier, it occurs at all strain rates. This is however not the case for subsequent slips along the 
same planes, which would also lead to the annihilation of the stacking faults, as a different process (leading to necking and failure, c.f. left panel of Fig. 9) becomes spontaneous first. At slow enough strain rates, this second round of slip restores the wire to its initial FCC structure (center panel of Fig. 9) and leads to a decrease of its width by one atomic layer. The process then repeats itself from this new pristine FCC structure. Unexpectedly, a process by which the FCC symmetry is broken in favor of an icosahedral tubular structure, shown in the right panel of Fig. 9, was then frequently observed. Once nucleated, this new phase grew by gradual conversion of the neighboring FCC regions, leading to elongation without failure up to very large strains. Such a process is consistent with experimental observations, where stretching at constant radius was observed once the wires became sufficiently thin [69].

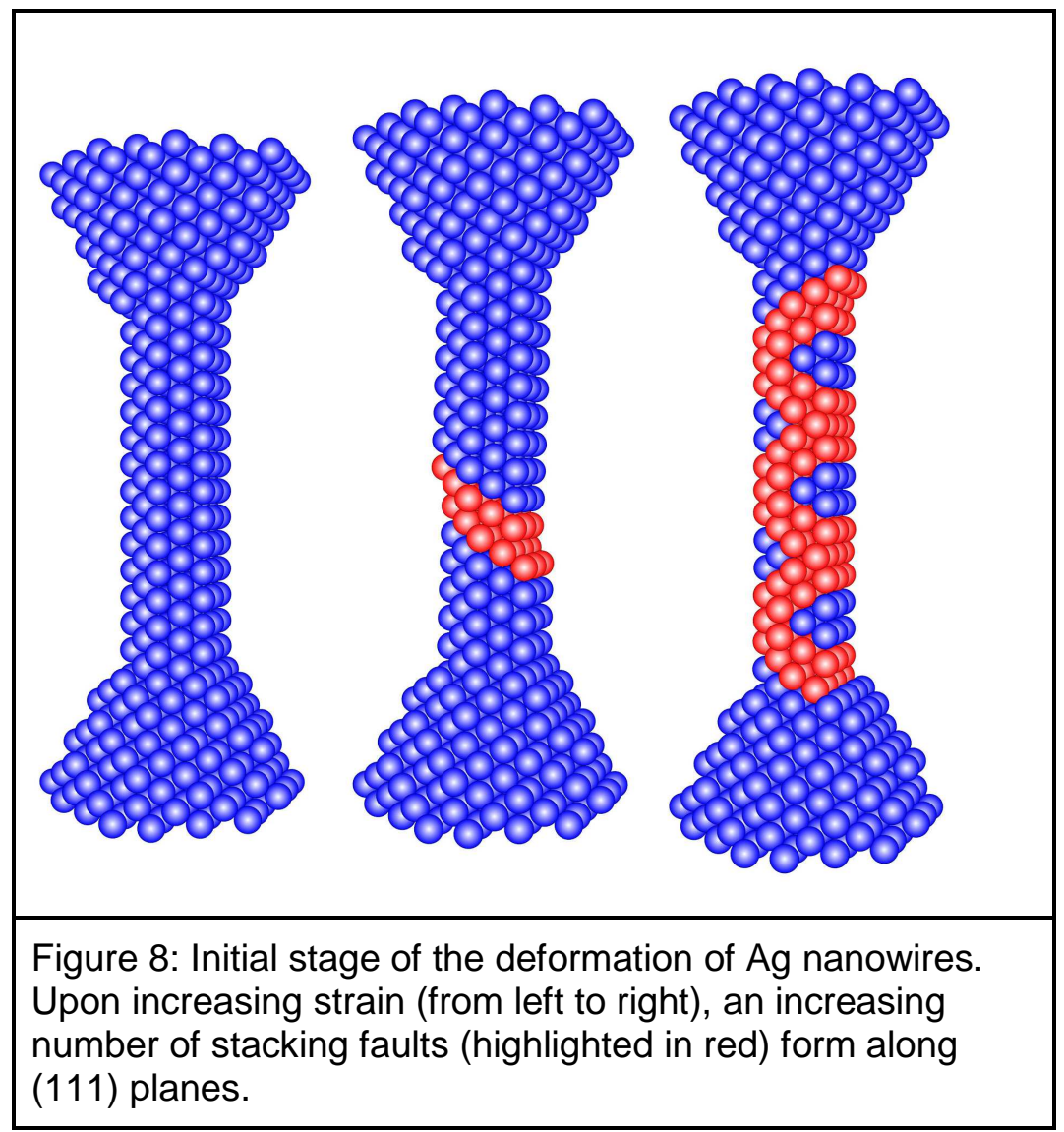

Through the use of Roadrunner, strain rates as low as $10^{3} \mathrm{~s}^{-1}$ were simulated (as compared to $\sim 10^{7} \mathrm{~s}^{-1}$ in conventional MD simulations), pushing simulation times into the ms. While these times are still short of the TEM experimental timescales, the use of ParRep gave access to an extremely wide dynamical range with which to explore the strain-rate sensitivity of the deformation. The largest simulation employed the totality of Roadrunner, and used about 12,000 replicas each parallelized over 10 cores [70]. In the initial phase of the deformation, slip proved to be infrequent enough that the parallel efficiency was above $90 \%$, corresponding to more than a microsecond of simulated time per minute of wall-clock time. As more complex defects formed, lower barriers appeared and the simulations were scaled back to about 1,000 replicas, 
still using 10 cores each. These results would have been difficult to obtain with other acceleration methods. For example, post-simulation analysis demonstrated the presence of extremely low transition rate prefactors, which would severely limit the performance of temperature accelerated dynamics (TAD), or even lead to inaccuracies if left unnoticed. Further, the displacement associated with a (111) slip is very small, which implies that a safe bias potential for hyperdynamics, e.g., in conjunction with the bond-boost approach, would have yielded only modest acceleration.

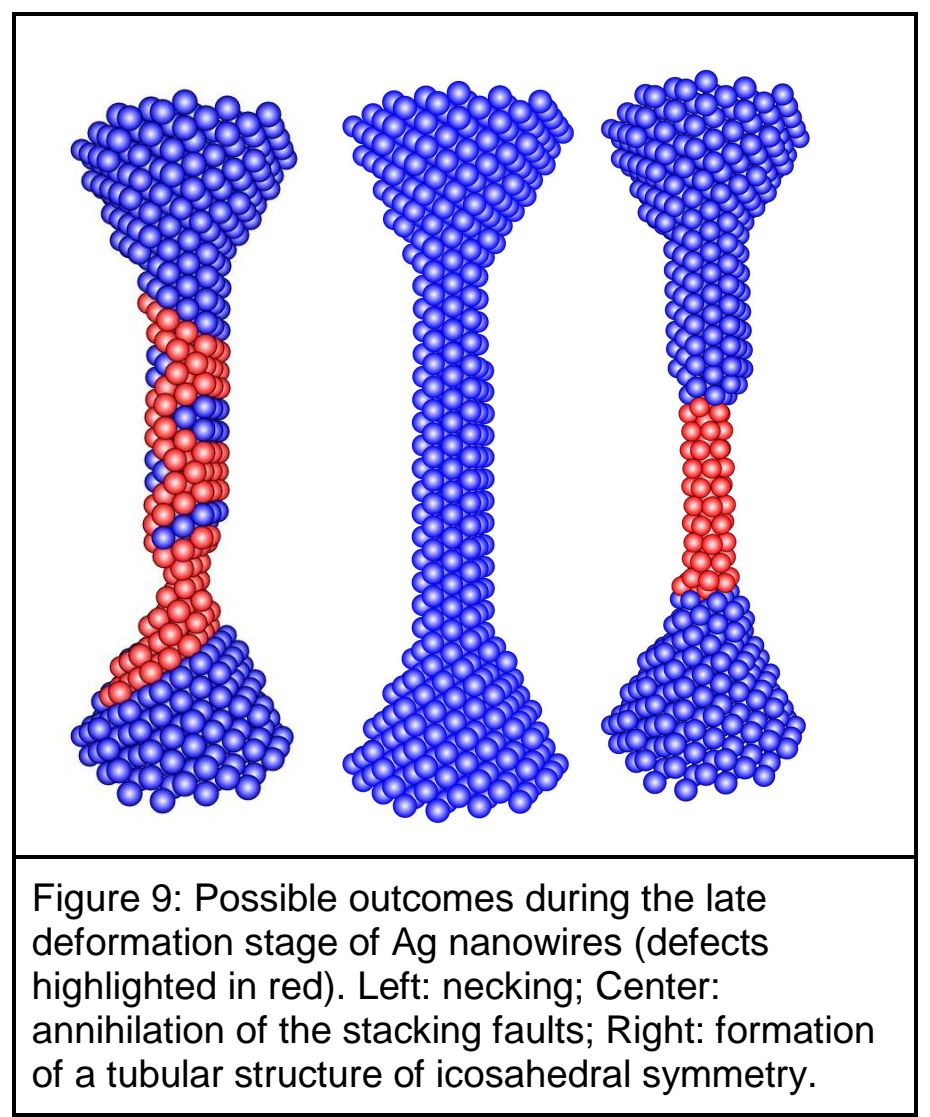

\section{Generalized definitions of states}

While the examples above show the power of associating each energy basin with a single state, it has long been recognized that ParRep can sometimes benefit from a more general state definition that is adapted to the problem at hand. Even before the formalization in terms of QSDs, this procedure was understood as being valid as long as states could be defined such that escape statistics were approximately Markovian. The generic strategy is to identify good "slow" degrees of freedom, i.e., degrees of freedom that can be used to flag the occurrence of the slow rate-limiting processes while being insensitive to fast ones. 
The first example of such a generalized state definition was reported in [71], where ParRep was used to study the pyrolysis of n-hexadecane at high temperatures. Given the very low barriers associated with conformational changes in the molecules compared to the barriers for bond breaking, the traditional definition of states would have led to extremely poor performance. In this case however, the small magnitude of the conformational barriers was a blessing, as they could then be ignored altogether when defining states: only the connectivity of the bonds was taken into account and all the configurations corresponding to the same set of isomers were lumped into the same state. This definition led to a sufficiently large separation of timescales, given the high barriers associated with bond breaking, and hence to almost Markovian kinetics. This approach was also used in Ref. [72].

A similar situation arises in the simulation of solid surfaces in contact with a liquid phase: transitions between basins in the liquid occur on extremely short, sub-ps, timescales compared to transitions involving solid atoms. However, sufficiently far from freezing, the liquid can be considered to quickly achieve "equilibrium" with respect to the current state of the solid surface. Therefore, the liquid atoms can be ignored when declaring a transition and only the positions of the solid atoms are used in deciding whether a transition occurred [42]. In other words, states were defined as containing all configurations that share a common topology of the solid-solid bonds. Such a strategy should be applicable to other cases where degrees of freedom can be partitioned into "fast" and "slow" subsets based on physical arguments [73].

Another limitation of the basin-based approach is the assumption that overcoming an energy barrier is the rate limiting step so that one can use energy minimizations (or quenching) to define reference configurations. While this assumption is usually valid in hard materials, it can fail for entropically stabilized systems. For example, it is known that the high temperature BCC phase of group IV metals is in fact unstable (in contrast to metastable) at zero temperature [74]. This means that there is no energy minimum associated with the BCC phase, even though it is dynamically stable at sufficiently high temperatures. Quenching a high temperature trajectory would lead to a collapse into a (defective) HCP structure. A similar situation was recently encountered in a study of the growth of nanoscale voids in uniaxially-strained $\mathrm{Cu}$ [75]. In this case, it was observed that voids can be dynamically stable for very long times at strains formally exceeding their mechanical instability limit. In this regime, quenching led to spontaneous growth of the nanovoids, even when the MD trajectories themselves showed no sign of such an event. Further investigation revealed that the softening of phonons under tensile strain led to the entropic stabilization of the voids. In this specific case, a reliable signature that the voids actually started growing was the abrupt decrease of the potential energy of the system due to the release of the large amount of elastic energy stored in the sample, and thus, a simple potential energy threshold was used to detect transitions. For this kind of system, one could also envision using finite-temperature averaged positions as the reference configuration; transitions would then be signaled by a persistent deviation away from this reference. This case provides a good example of a system that would have been difficult to simulate using other methods. For example, TAD would have failed due to the assumption underlying harmonic TST that energy barriers are the dynamical bottlenecks. For the same reasons, AKMC techniques would have 
also failed. While formally applicable, it would have been difficult to design a safe bias potential in hyperdynamics, as the location of the free energy barrier is not a priori clear.

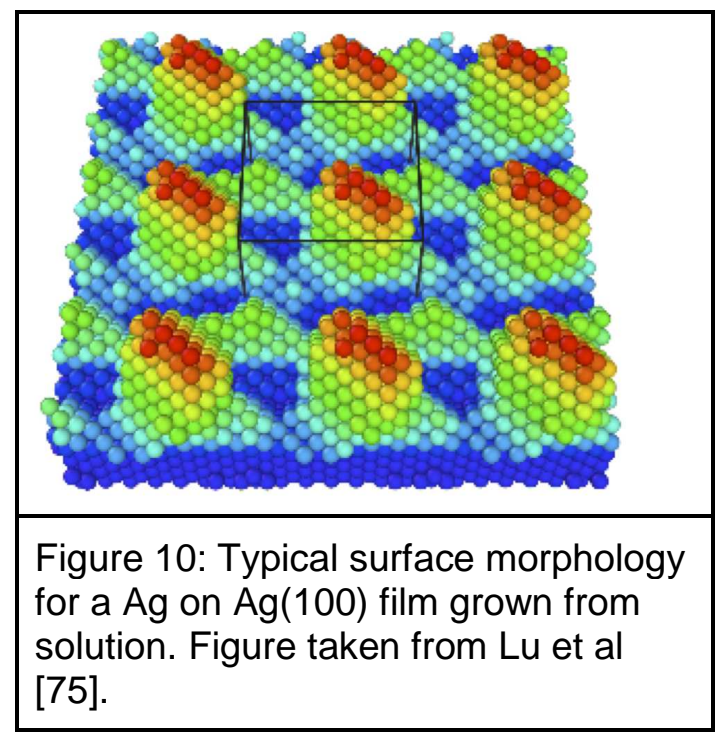

Properly defining states can prove crucial to extending the range of applicability of ParRep to new classes of systems. For example, what if one now wanted to simulate the growth of a solid through precipitation of solute atoms from a liquid solution? As discussed above, the dynamics of the solid atoms themselves can be accelerated using ParRep. However, at first glance, it would appear that properly accelerating the deposition of new atoms onto the surface would be beyond the scope of ParRep, as diffusive, Brownian-like, motion in solution is not a rare-event process. The key is to realize that one does not need to accelerate diffusion, but only deposition, as the precise location of every solute atom is inconsequential for the growth of the solid; one only needs to know when and where the next solute atom will stick onto the surface [76]. In that context, one can show that, at large times, the concentration of solute atoms in solution will approach the QSD of a Smoluchowski equation with absorbing boundary conditions at the solid surface. Therefore, in that regime, deposition of a new atom is a rare event that can be accelerated by ParRep insofar as generating independent samples from the solute QSD on each replica is integrated into the dephasing stage. This process can be sped up by using continuum solvers coupled with the Fleming-Viot algorithm [77]. Together with a spatiallymultiscale representation of the solution, this approach enabled the long-time simulation of the growth of a Ag film in contact with a very thick liquid film [76]. Through these simulations, running at around $75 \%$ parallel efficiency on 200 replicas, it was observed that the diffusive nature of the solutes' motion resulted in an increased roughness of the film due to strong steering effects. A typical morphology of the surface is shown in Fig. 10. We believe that the range of problems amenable to acceleration with ParRep can be greatly extended through judicious definitions of states. 


\section{Tricks of the Trade}

Through simple reorganizations of algorithm and bookkeeping, ParRep implementations used in practice can often be significantly more efficient than the naive version illustrated in Fig. 2. Some of these possible improvements are highlighted in this section.

The most obvious source of inefficiency in the naive implementation is that $N_{r}-1$ replicas remain idle during decorrelation. However, given a state with a sufficiently large spectral gap, most of the trajectories will end the decorrelation stage in the same state where they began. Therefore, one might speculate that no transition will occur during decorrelation and initiate dephasing on the other replicas right away, hence overlapping both stages. If this assumption proves wrong, then dephasing is carried out again from the proper state. This simple trick can decrease the amount of overhead by up to a factor of two when follow up transitions are rare.

The dominant remaining source of overhead is dephasing. Contrary to the Boltzmann distribution, the QSD depends on the details of the dynamics, such as the specific value of the Langevin friction. Thus, one cannot directly tap into the considerable arsenal of thermodynamic sampling methods in order to improve the efficiency of dephasing. In fact, at this time, we know of no other method that would be more efficient than the rejection-based approach discussed above, while remaining as accurate. Pending the development of such a method, the alternative is to maximize the practical use of each successful dephasing stage. One way to do so is to realize that, after each ParRep cycle, $N_{r}-1$ replicas (excluding the one on which the transition occurred first) still possess a good sample from the QSD for the current state, as, by definition, they ran for a time longer than $\tau_{c}$ without escaping. In the event that the ParRep trajectory revisits this state (and of course, assuming that this revisit can be detected), these replicas don't have to dephase once more, as they can instead reuse their last configuration in that state. On its own, this bookkeeping trick significantly alleviates the low-barrier problem when these barriers connect a relatively small set of states that will hence be revisited many times before the trajectory moves on to a different region of configuration space.

Note that in cases where performance is not limited by the physics of the system of interest but by the availability of massively parallel computing resources, i.e., when $N^{*} \gg N_{r}$, ParRep can be combined with other AMD methods, such as hyperdynamics [30], in order to bridge this gap [78]. Such a combination provides a multiplicative increase of the boost, provided none of the methods taken alone has already saturated the available acceleration.

Finally, it is important to keep in mind that the practical advantage of ParRep over spatiallyparallelized MD relies on the fact that the latter does not scale well for small systems. If it did, one would definitely opt for this solution, as it would be exact and free of the overhead discussed above. That being said, these two approaches do not have to be exclusive. One should first spatially parallelize their MD engine up to the point where the efficiency starts to 
drop. Only then would one introduce ParRep in order to enable scaling to even larger numbers of processors. Indeed, it is always beneficial to keep the number of replicas to a minimum as this minimizes the fraction of effort dedicated to dephasing. While little might be gained by such a hierarchical approach for very small systems (hundreds of atoms), the benefits can be significant for systems containing thousands of atoms or more.

For especially large systems, a new scaling issue emerges. Assuming that there is a typical rate at which transitions occur per volume of material (this is not necessarily the case, but is often true), then as the system is made larger, the average time between events becomes shorter and the overall potential ParRep boost drops in a way that is roughly inversely proportional to system size. For systems in which the transition events are spatially local, then the overall parallel efficiency can be raised substantially by combining ParRep with the synchronous sublattice (SL) method of Shim and Amar [79], originally proposed for spatial parallelization of kinetic Monte Carlo. In the SL method, the system is spatially decomposed, and then each spatial domain is further decomposed into sublattice regions. At any time, dynamics are advanced on only one sublattice region in each domain, so that every dynamically active region is buffered from other dynamically active regions by dormant sublattice regions. Cycling through these sublattices, keeping the time for active dynamics on each sublattice (the cycle time) short, gives controllably accurate dynamics. The advantage over other ways to parallelize KMC is that it eliminates the need to explicitly correct for boundary conflicts in which events may happen in the wrong time order. This SL approach is also powerful for spatially parallelizing AMD dynamics [80,81], because they too are event-based. Combining ParRep with the SL method offers a way to parallelize on three levels: At the lattice/sublattice level (as in the SL-KMC), at the ParRep level (because an independent ParRep simulation is performed in each sublattice domain, and at the MD level (because the force call for each ParRep replica can be parallelized, as discussed in the paragraph above). The result is that this SL-ParRep method [81], at the expense of assuming that transitions never exceed a certain spatial extent (the size of the subdomain), gives a greater total simulation speed than direct MD, parallelized MD, ParRep, or ParRep with parallelized MD for each replica, for certain regimes in the space of system size, number of processors, and average transition rate per volume of material. For example, for million-atom systems with an average escape rate of $10^{6}$ per second per atom, this SL-ParRep approach is expected to be more than an order of magnitude faster than any of these other methods on a machine with a processor count of $10^{7}$ or higher [81].

\section{Conclusion}

As demonstrated above, ParRep is an extremely general approach. The only requirement is that the dynamics, conditioned on having remained in one state, approach a QSD at long times. If this assumption is verified, ParRep can generate trajectories with arbitrary accuracy, no matter the source of the metastability. Indeed, one does not need to assume anything about the nature of the dynamical bottlenecks --- they could be energetic or entropic --- nor does one need to invoke approximate rate theory, e.g., transition state theory as in hyperdynamics, or the harmonic approximation to TST, as in TAD. ParRep can be translated to continuous or discrete 
settings and is not limited to atomistics; it can potentially be used on any dynamical system where the long time evolution proceeds through a sequence of rare events. ParRep is algorithmically simple and offers a rigorous strategy for the time-wise parallelization of the generation of trajectories, offering a simple option to leverage increasingly common massivelyparallel platforms. As it matures into adulthood, we expect ParRep to become an essential and standard tool in the computational material scientist's toolbox.

\section{Acknowledgements}

We are grateful to our collaborators Yalin Dong, Timothy Germann, Tony Lelievre, Claude Le Bris, Albert Lu, Sheng-Niang Luo, Mitchell Luskin, Enrique Martinez, Ashlie Martini, Chun-Wei Pao, Steven Stuart, and Sriram Swaminarayan. This work was supported by the United States Department of Energy (U.S. DOE), Office of Science, Office of Basic Energy Sciences, Materials Sciences and Engineering Division and also reviews work supported by the Los Alamos National Laboratory (LANL) LDRD program. LANL is operated by Los Alamos National Security, LLC, for the National Nuclear Security Administration of the U.S. DOE, under contract DE-AC52-O6NA25396.

\section{References}

[1] J.B. Gibson, A.N. Goland, M. Milgram, G.H. Vineyard, Physical Review 120, 1229 (1960).

[2] M.S. Daw and M.I. Baskes, Phys. Rev. B 29, 6443 (1984).

[3] D.E. Shaw, et al., Communications of the ACM 51, 91 (2008).

[4] A.F. Voter, in Radiation Effects in Solids, K.E. Sickafus,

E.A. Kotomin, and B.P. Uberuaga, editors (Springer, NATO Publishing Unit, Dordrecht, The Netherlands, 2006), pp. 1-24.

[5] A.B. Bortz, M.H. Kalos, and J.L. Lebowitz, J. Comp. Phys. 17, 10 (1975).

[6] D.T. Gillespie, J. Comp. Phys. 22, 403 (1976).

[7] R. Marcelin, Ann. Physique 3,120 (1915).

[8] E. Wigner, Z. Phys. Chem. B19, 203 (1932).

[9] H. Eyring, J. Chem. Phys. 3, 107 (1935).

[10] G.H. Vineyard, J. Phys. Chem. Solids 3, 121 (1957).

[11] D. Chandler, J. Chem. Phys. 68, 2959 (1978).

[12] A.F. Voter and J.D. Doll, J. Chem. Phys. 82, 80 (1985).

[13] P.J. Feibelman, Phys. Rev. Lett. 65, 729 (1990).

[14] G.L. Kellogg and P.J. Feibelman, Phys. Rev. Lett. 64, 3143 (1990).

[15] C. Chen and T.T. Tsong, Phys. Rev. Lett. 64, 3147 (1990).

[16] C.L. Liu and J.B. Adams, Surf. Sci. 268, 73 (1992).

[17] R. Wang and K.A.Fichthorn, Molec. Sim. 11, 105 (1993).

[18] J.C. Hamilton, M.S.Daw, and S.M.Foiles, Phys. Rev. Lett. 74, 2760 (1995).

[19] G. Henkelman and H. Jonsson, J. Chem. Phys. 111, 7010 (1999).

[20] J. Marian, B.D. Wirth, and J.M. Perlado, Phys. Rev. Lett. 88, 255507 (2002). 
[21] B.P. Uberuaga, R. Smith, A.R. Cleave, F. Montalenti, G. Henkelman, R.W. Grimes, A.F. Voter, and K.E. Sickafus, Phys. Rev. Lett. 92, 115505 (2004).

[22] Y.A. Du, R.G. Hennig, and J.W. Wilkins, Phys. Rev. B 73, 5 (2006).

[23] B.P. Uberuaga, R.G. Hoagland, A.F. Voter, and S.M. Valone, Phys. Rev. Lett. 99, 135501 (2007).

[24] X.-M. Bai, A.F. Voter, R.G. Hoagland, M. Nastasi, and B.P. Uberuaga, Science 327, 1631 (2010).

[25] D.A. Andersson, F.J. Espinosa-Faller, B.P. Uberuaga and S.D. Conradson, J. Chem. Phys. 136, 234702 (2012).

[26] G. Henkelman and H. Jonsson, J. Chem. Phys. 115, 9657 (2001).

[27] T. Middleton and D.J. Wales, J. Chem. Phys. 120, 8134 (2004).

[28] F. El-Mellouhi, N. Mousseau, and L.J. Lewis, Phys. Rev. B 78, 153202 (2008).

[29] L. Xu and G. Henkelman, J. Chem. Phys. 129, 114104 (2008).

[30] A.F. Voter, J. Chem. Phys. 106, 4665 (1997).

[31] A.F. Voter, Phys. Rev. B 57, 13985 (1998).

[32] M.R. Sörensen and A.F. Voter, J. Chem. Phys. 112, 9599 (2000).

[33] C.-Y. Lu, D.E. Makarov, and G. Henkelman, J. Chem. Phys. 133, 201101 (2010).

[34] A.F. Voter, Phys. Rev. Lett. 78, 3908 (1997).

[35] R.A. Miron and K.A. Fichthorn, J. Chem. Phys 119, 6210 (2003).

[36] F. Montalenti and A.F. Voter, J. Chem. Phys. 116, 4819 (2002).

[37] B.P. Uberuaga, R. Smith, A.R. Cleave, G. Henkelman, R.W. Grimes, A.F. Voter, and K.E. Sickafus, Phys. Rev. B, 71, 104102 (2005).

[38] D.G. Tsalikis, N. Lempesis, G.C. Boulougouris, and D.N. Theodorou, J. Phys. Chem. B 114, 7844-7853 (2010).

[39] Y. Shim and J.G. Amar, J. Chem. Phys. 134, 054127 (2011).

[40] V. Bochenkov, N. Suetin, and S. Shankar J. Chem. Phys. 141, 094105 (2014).

[41] B.P. Uberuaga and A.F. Voter, in Radiation Effects in Solids, edited by K. E. Sickafus, E. A. Kotomin and B.P. Uberuaga (Springer, NATO Publishing Unit, Dordrecht, The Netherlands, (2006) pp. 25-43.

[42] D. Perez, B.P. Uberuaga,Y. Shim, Jacques G. Amar, and Arthur F. Voter, Ann. Rep. Comp. Chem. 5, 79 (2009).

[43] http://lammps.sandia.gov

[44] http://theory.cm.utexas.edu/eon/

[45] http://www.ccp5.ac.uk/DL POLY CLASSIC/

[46] C. Sminchisescu and B. Triggs, Image and Vision Computing 24, 279 (2006).

[47] C. Le Bris, T. Lelièvre, M. Luskin, and D. Perez, Monte Carlo Methods and Applications 18, 119 (2012).

[48] MPI: A Message-Passing Interface Standard Version 3.0, Message Passing Interface Forum, http://www.mpi-forum.org (2012).

[49] M. Kijima, Markov Processes for Stochastic Modeling, Chapman \& Hall, London (1997).

[50] B.P. Uberuaga, M. Anghel, and A.F. Voter, J. Chem. Phys. 120, 6363 (2004).

[51] B. Puchala, M.L. Falk, and K. Garikipati, J. Chem. Phys. 132, 134104 (2010).

[52] J.D. Chodera, N. Singhal, V.S. Pande, K.A. Dill, and W.C. Swope, J. Chem. Phys. 126, 155101 (2007). 
[53] F. Noé, J. Chem. Phys. 128, 244103 (2008).

[54] P. Metzner, M. Weber, and C. Schütte, Phys. Rev. E 82, 031114 (2010).

[55] F. Noé and F. Nüske, Multiscale Modeling \& Simulation 11, 635 (2013).

[56] Andrew Binder, Tony Lelièvre, Gideon Simpson, arXiv:1404.6191 (2014).

[57] E. Meerbach, J. Latorre, and C. Schütte, Multiscale Modeling \& Simulation 10, 1263 (2012).

[58] B.P. Uberuaga, S. Stuart, and A.F. Voter, Phys. Rev. B 75, 014301 (2007).

[59] B.P. Uberuaga, A.F. Voter, K.K. Sieber, and D.S. Sholl, Phys. Rev. Lett. 91, 105901 (2003).

[60] O. Kum, B.M. Dickson, S.J. Stuart, B.P. Uberuaga, and A.F. Voter, J. Chem. Phys. 121, 9808 (2004).

[61] Y. Mishin, A. Suzuki, B.P. Uberuaga and A.F. Voter, Phys. Rev. B 75, 224101, (2007).

[62] A. Martini, Y. Dong, D. Perez, and A.F. Voter, Tribol. Lett. 36, 63 (2009).

[63] Y. Dong, D. Perez, A.F. Voter, and A. Martini, Tribol. Lett. 42, 99 (2011).

[64] Y. Duan, J.W. Halley, L. Curtiss, and P. Redfern, J. Chem. Phys. 122, 054702 (2005).

[65] D.H. Warner, W.A. Curtin, and S. Qu, Nature Materials 6, 877 (2007).

[66] B. Zagrovic, E.J. Sorin, and V.S. Pande, J. Mol. Biol. 313, 151 (2001).

[67] Q. Li, Y. Dong, D. Perez, A. Martini, R. Carpick, Phys. Rev. Lett. 106, 126101 (2011).

[68] A. Martini, Y. Dong, D. Perez, A.F. Voter, Tribo. Lett. 36, 63 (2009).

[69] V. Rodrigues, J. Bettini, A.R. Rocha, L.G.C. Rego, D. Ugarte, Phys. Rev. B 65153402 (2002).

[70] S. Swaminarayan, in Contemporary High Performance Computing: From Petascale toward Exascale, edited by Jeffrey S. Vetter, Chapman \& Hall/CRC computational science series (2012).

[71] O. Kum, B.M. Dickson, S. Stuart, B.P. Uberuaga, and A.F. Voter, J. Chem. Phys. 121, 9808 (2004).

[72] K.L. Joshi, S. Raman, and A.C.T. van Duin, J. Phys. Chem. Lett. 4, 3792 (2013).

[73] O. Uche, D. Perez, A.F. Voter, and J.C. Hamilton, Phys. Rev. Lett. 103, 046101 (2009).

[74] P. Souvatzis, O. Eriksson, M.I. Katsnelson, and S.P. Rudin, Phys. Rev. Lett. 100, 095901 (2008).

[75] D. Perez, S. N. Luo, A. F. Voter, and T. C Germann, Phys. Rev. Lett. 110, 206001 (2013)

[76] C.Y. Lu, A.F. Voter, and D. Perez, J. Chem. Phys. 140, 044116 (2014).

[77] P. Ferrari and N. Maric, Electron. J. Probab. 12, 684 (2007)

[78] A.F. Voter and T.C. Germann, Mat. Res. Soc. Symp. Proc. 528, 221 (1998).

[79] Y. Shim and J.G. Amar, Phys. Rev. B 71, 125432 (2005).

[80] Y. Shim, J.G. Amar, B.P. Uberuaga, and A.F. Voter, Phys. Rev. B 76, 205439 (2007).

[81] E. Martinez, B.P. Uberuaga, and A.F. Voter, Phys. Rev. E 89, 063308, (2014). 